\title{
Power-efficient beam tracking during connected mode DRX in mmWave and sub-THz systems
}

\author{
Syed Hashim Ali Shah, Student Member, IEEE, Sundar Aditya, Member, IEEE \\ and Sundeep Rangan, Fellow, IEEE
}

\begin{abstract}
Discontinuous reception (DRX), wherein a user equipment (UE) temporarily disables its receiver, is a critical power saving feature in modern cellular systems. DRX is likely to be aggressively used at mmWave and sub-THz frequencies due to the high front-end power consumption. A key challenge for DRX at these frequencies is blockage-induced link outages: a UE will likely need to track many directional links to ensure reliable multi-connectivity, thereby increasing the power consumption. In this paper, we explore bandit algorithms for link tracking in connected mode DRX that reduce power consumption by tracking only a fraction of the available links, but without adversely affecting the outage and throughput performance. Through detailed, system level simulations at $28 \mathrm{GHz}(5 \mathrm{G})$ and $140 \mathrm{GHz}$ (6G), we observe that even sub-optimal link tracking policies can achieve considerable power savings with relatively little degradation in outage and throughput performance, especially with digital beamforming at the UE. In particular, we show that it is feasible to reduce power consumption by $75 \%$ and still achieve up to $95 \%(80 \%)$ of the maximum throughput using digital beamforming at $28 \mathrm{GHz}(140 \mathrm{GHz})$, subject to an outage probability of at most $1 \%$.
\end{abstract}

\section{Index Terms}

Discontinuous reception (DRX), Multiple-play multi-armed bandits (MP-MAB), power-efficient beam tracking, sub-terahertz $(\mathrm{THz})$ communications, millimeter wave (mmWave) communications, 5G, $6 \mathrm{G}$.

The authors are with NYU WIRELESS, Tandon School of Engineering, New York University, Brooklyn, NY 11201, USA (Email: \{s.hashim, sundar.aditya, srangan\}@nyu.edu).

This work was supported by the National Science Foundation under Grants 1302336, 1564142, and 1547332, NIST, SRC and the industrial affiliates of NYU WIRELESS. A part of this paper was presented at the International Workshop on Signal Processing Advances in Wireless Communications (SPAWC), 2019 held at Cannes, France [1]. 


\section{INTRODUCTION}

\section{A. Motivation}

Mobile wireless communication in the mmWave and sub-THz bands enable multi-Gbps peak throughput, but at the cost of high power consumption in both the radio frequency front-end (RFFE) and digital baseband processing [2]-[4]. The high power consumption arises from the need to support a large number of antenna elements at very high sample rates, along with the relative inefficiency of RF components at high frequencies. Indeed, power consumption particularly for mobile devices - is one of the most significant challenges facing $5 \mathrm{G}$ deployments today. For example, power estimates in [5] show that peak mobile RFFE power consumption for a typical $28 \mathrm{GHz}$ device can exceed $1 \mathrm{~W}$ - a large portion of the total power budget. Recently, there has been significant interest in communication above $100 \mathrm{GHz}$, including the sub-THz and $\mathrm{THz}$ bands [6]-[9]. Power consumption issues are likely to become even more acute in these frequencies. For example, a recent power estimate [10] showed that the UE receiver for a New Radio (NR)-like system at $140 \mathrm{GHz}$ would require more than 30 times the power consumption of a receiver at $28 \mathrm{GHz}$, based on current device performance.

Discontinuous Reception (DRX) [11], [12], where a mobile device or UE temporarily disables its RFFE, is one of the most widely used tools to reduce power consumption in mobile devices. The DRX mechanism for the 5G new radio (NR) standards consists of three modes (states) [13] - Idle, Connected and Inactive - as opposed to legacy DRX with two states [14]. In this paper, we focus on connected mode DRX, whose operation is illustrated in Fig. 1.

Implementing DRX poses unique challenges in the mmWave and sub-THz bands [15], [17], [18]. Most importantly, mmWave systems communicate using narrow directional beams to overcome the high isotropic path loss [2]-[4]. Directional links need to be tracked to detect changes in the handset orientation, as well as link blockages - a key challenge in the mmWave bands [19][21]. In addition, mobile devices in mmWave cellular systems will likely require maintaining links to multiple cells for macro-diversity [22]. Thus, UEs will likely need to track links from multiple directions from multiple cells during the Beam measurements and Feedback segments shown in the left panel of Fig. 1. This link tracking reduces the time a UE can turn off its RFFE, thereby creating trade-offs between power consumption, directional tracking and link reliability. For instance, if the UE decides to reduce link tracking to save power (right panel of Fig. 1), how 


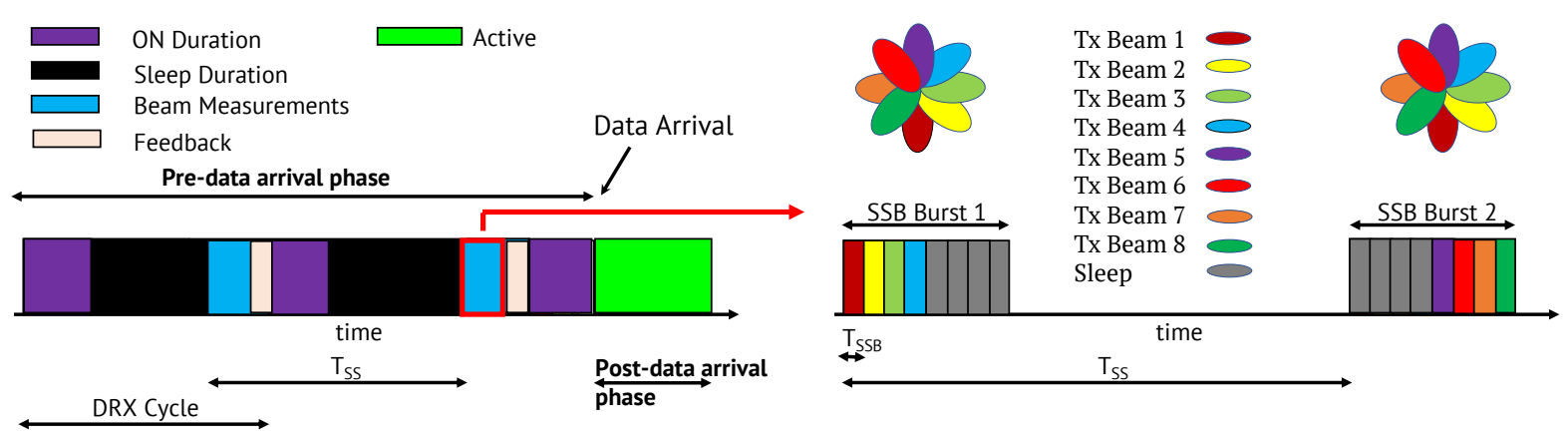

Fig. 1: Left Panel: UE in connected mode DRX with beam tracking [15], [16]. Right Panel: Magnified time line of a TX beam sweep using SSBs at the gNB, with $N_{\mathrm{SSB}}=8$. The UE is awake for $K=4$ SSB time slots in each SSB burst period. If the UE employs digital beamforming, then it can track along all the RX directions in each SSB time slot that it is awake for. With analog beamforming, however, the UE can only track along a single RX direction in a given SSB time slot.

should the UE track the links so that the UE performance (e.g., outage probability, throughput, etc.) does not suffer severely? As we will show below, the number of beams to track and the rate of blockages increases in the bands above $100 \mathrm{GHz}$ making the tradeoff even more important in the sub-THz regime.

In this paper, we address this question using a two-step approach, where, (i) given a constraint, $K$, which is related to the number of links to track by a scaling factor (and hence, acts as a power constraint), we represent the choice of links to track over time as the outcome of a feasible policy for a multiple-play multi-armed bandit (MP-MAB) problem; and, (ii) given a policy for the MP-MAB problem in (i), we then identify the smallest $K$ as the solution to an optimization problem that captures the power-performance trade-off.

Our contributions in this paper are:

1) For connected mode DRX in 3GPP NR, we estimate the UE RFFE power consumption at carrier frequencies of 28 and $140 \mathrm{GHz}$ with analog and digital beamforming architectures, assuming system parameters taken from the 3GPP standard ${ }^{1}$. We show that the directional link tracking measurements are responsible for most of the power consumed in connected

\footnotetext{
${ }^{1}$ We use 3GPP NR as a benchmark, since it is the dominant standard for 5G systems. However, our DRX analysis would likely apply to other directional systems with intermittent transmissions as well.
} 
mode DRX, especially when the UE tracks the maximum permitted number of links.

2) To reduce power consumption in connected mode DRX, we choose to track only a subset of the links (depending on $K$ ) at any time. We then cast the choice of links to track as the outcome of a policy for a MP-MAB problem. Then, for a given a policy, we identify the smallest $K$ as the solution to an optimization problem that captures the power-performance trade-off.

3) Since it is hard to obtain a statistical characterization of the effects of multipath, mobility and correlated blockages on the link SNRs, it is difficult to formulate a link tracking policy with provable performance guarantees (e.g. sub-linear regret). Hence, we consider four suboptimal policies and compare their performance using detailed system-level simulations based on the 3GPP NR standards at 28 and $140 \mathrm{GHz}$, which are representative of a 5G mmWave [23] and a hypothetical 6G sub-THz [8] operating environment, respectively, for both analog and digital beamforming at the UE. While the question of a suitable UE beamforming architecture depends on many factors, such as spectral efficiency, hardware complexity, etc., our focus in this paper is restricted to the RFFE power consumption and link tracking capabilities of each architecture. For each combination of carrier frequency and beamforming architecture, the winning policy (i.e., the best performing policy among our limited selection in our simulations) provides useful performance benchmarks that a well-designed policy can be expected to satisfy.

4) From the winning policies in our simulations, we conclude that a well-designed link tracking policy can realize at least $50 \%$ power savings and achieve $85 \%$ of the maximum throughput with at most $1 \%$ outage probability in a $5 \mathrm{G} \mathrm{mmWave} \mathrm{environment} \mathrm{at} 28 \mathrm{GHz}$ with analog beamforming at the UE. With digital beamforming in the same environment, more power savings (at least 75\%) and a larger fraction (95\%) of the maximum throughput can be achieved for the same constraint on the outage probability. At $140 \mathrm{GHz}$ with analog beamforming, none of our policies achieves an outage probability below 1\%, as the UE is constrained to track only a small fraction of the total number of links, even at maximum power consumption. This finding highlights the need for standardization efforts at $140 \mathrm{GHz}$ for analog beamforming to be viable, both in terms of power efficiency and performance. On the other hand, digital beamforming in the same environment can save $75 \%$ power, while achieving $80 \%$ of the maximum throughput for an outage probability of at most $1 \%$. 


\section{B. Related Work}

DRX for LTE systems was studied in [24], while [15], [17], [18] focused on directional DRX for mmWave systems. These works concentrate on optimizing DRX parameters in the MAC/Radio Resource Control (RRC) layer (e.g., ON Timer, DRX cycle, etc.), whereas in this paper, we focus on the beam management aspect of DRX, which is primarily a physical (PHY) layer issue. Furthermore, [15], [17], [18] also focus on DRX performance during the data arrival phase (i.e., when there are data packets for the UE on the downlink), through metrics like the queuing delay, and the wake-up latency. In contrast, we focus on the pre-data arrival phase (i.e., no data arriving on the downlink), where we are interested in the outage probability, since an outage would lead to a loss of connectivity to the network.

DRX in a multi-connectivity setting is addressed in [25], but for the relatively simple scenario of dual connectivity in LTE systems, where the links are not prone to blocking-induced outages. However, at mmWave and sub-THz frequencies, where blockages are a major impediment, the degree of multi-connectivity is an important system parameter that impacts both the UE performance as well as power consumption, especially at sub- $\mathrm{THz}$ frequencies where the frequency and severity of blockages are likely to be greater than that at mmWave. To the best of our knowledge, this power-performance trade-off and its implications for sub-THz UE beamforming architecture, which is a key theme of this paper, has not been studied previously.

Beam management has been extensively studied for mmWave systems in [26]-[29]. However, these works focus on beam tracking during data transfer and do not consider the power efficiency of link tracking mechanisms. In terms of scope, the work closest to ours is [16], where connected mode DRX is investigated in the pre-data arrival phase, but in a very simple mobile mmWave environment with only analog beamforming deployed at the UE. Crucially, it does not address the power versus performance trade-off associated with tracking multiple links at the UE. In this paper, we investigate this trade-off using detailed system-level simulations based on the 3GPP NR standards, for a $5 \mathrm{G}$ mmWave system at $28 \mathrm{GHz}$ and a hypothetical $6 \mathrm{G}$ sub-THz system at $140 \mathrm{GHz}$, with both analog and digital beamforming at the UE. To the best of our knowledge, this is the first work to investigate the power efficiency of beam management, especially for sub-THz systems. 


\section{Organization}

This paper consists of seven sections. In Section II, we provide an overview of connected mode DRX, along with a model for DRX power consumption. In Section III, we capture the trade-off between power consumption and the measured channel quality in connected mode DRX by formulating the choice of links to track as the outcome of a policy for a MP-MAB problem. In Section IV, we present four sub-optimal, but effective, policies for the MP-MAB problem in Section III. The details of our simulation setup, modeling a $5 \mathrm{G} \mathrm{mmWave} \mathrm{system} \mathrm{at} 28 \mathrm{GHz}$ and a hypothetical 6G sub-THz system at $140 \mathrm{GHz}$, are presented in Section V. Simulation results capturing the power-performance trade-off for the policies in Section IV are presented in Section VI, culminating in a discussion on the signficance of our results. Finally, Section VII concludes the paper with a summary.

\section{Notation}

Throughout this paper, vectors and matrices are represented by lower and upper case boldface letters, respectively. All vectors are column vectors, 1 denotes the all-one vector, and $(.)^{T}$ and $(.)^{H}$ denote the transpose and Hermitian operators, respectively. $\mathbb{R}$ and $\mathbb{C}$ respectively denote the set of real and complex numbers, $\mathbb{P}($.$) denotes probability, \mathbb{E}[$.$] the expectation operator, \mathcal{U}[a, b]$ a uniform random variable over $[a, b]$, and $\mathcal{N}\left(\mu, \sigma^{2}\right)$ a normal random variable with mean $\mu$ and variance $\sigma^{2}$. $\operatorname{Gamma}(a, b)$ and $\operatorname{Beta}(a, b)$ respectively denote a gamma and a beta random variable, whose probability distribution functions (pdfs) $f_{\Gamma}($.$) and f_{\text {Beta }}($.$) are given by:$

$$
\begin{aligned}
f_{\text {Gamma }}(y) & =\frac{1}{\Gamma(a) b^{a}} y^{a-1} \exp (-y / b), y \geq 0 \\
f_{\text {Beta }}(y) & =\frac{y^{a-1}(1-y)^{b-1}}{B(a, b)}, y \in[0,1]
\end{aligned}
$$

where $\Gamma($.$) and B(.,$.$) denote the gamma and beta functions, respectively. For integers a_{0}$ and $b_{0}\left(b_{0} \neq 0\right),\left\lfloor a_{0} / b_{0}\right\rfloor$ and $\left(a_{0} \bmod b_{0}\right)$ denote the quotient and remainder of $a_{0} / b_{0}$, respectively.

\section{AnAlysis of 3GPP Connected Mode DRX}

We first present an overview of connected mode DRX in the 3GPP NR standard [13]. Consider a UE situated within the coverage area of $N_{\text {cell }}$ gNBs (base stations). Let $N_{\mathrm{TX}}$ and $N_{\mathrm{RX}}$ denote 
the number of antenna elements at the gNB and the UE, respectively. A key aspect of mmWave and sub-THz communications is the use of beamforming. We will assume that the $\mathrm{gNB}$ and the UE transmit and receive using finite beamforming codebooks [30], [31] for channel tracking and synchronization. Without loss of generality, we assume that the codebook size at the gNB and the UE equals $N_{\mathrm{TX}}$ and $N_{\mathrm{RX}}$, respectively, which correspond to one codeword for each orthogonal spatial degree of freedom ${ }^{2}$.

\section{A. Connected Mode DRX concepts overview}

Beam Management in Connected Mode DRX: DRX is a MAC layer procedure, which determines when the UE goes to sleep (i.e., turns off its RFFE) to save power. In connected mode DRX, the UE wakes up periodically for the following tasks:

(a) Beam management (represented by the Beam Measurements and Feedback segments in the left panel of Fig. 1), which determines the link(s) through which the UE maintains connectivity to the network, and

(b) to receive notifications through at least one link in (a) of data arrival on the downlink (represented by the ON Duration segment in Fig. 1).

In this paper, we focus on (a) alone, drawing attention to the power intensive nature of beam management process, which undermines the effectiveness of DRX as a power-saving mechanism if the UE tracks all the permitted links ${ }^{3}$. To reduce power consumption due to beam management, we consider policies that identify a subset of 'good' links to track, without jeopardizing the UE's ability to receive any incoming data in step (b) above. However, to study the evolution of our policies with changing link conditions, we assume that the UE is in the pre-data arrival phase throughout (i.e., there is no data arriving on the downlink), so that the UE continues to remain in connected mode DRX and beam management happens periodically without being interrupted by the UE going into Active state.

Directional Tracking using Synchronization signals: During beam management, we assume that the UE tracks the directional channel quality from the cells via the 5G NR synchronization

\footnotetext{
${ }^{2}$ A smaller codebook size reduces beam training overhead at the expense of beamforming resolution.

${ }^{3}$ The set of links a UE is permitted to track in each DRX cycle in Fig. 1 depends on the beamforming scheme at the UE and is, in general, smaller than the total number of available links. See (3) and Remark 4 for more details.
} 
signal blocks (SSBs) [13]. In the 5G NR system, each gNB periodically transmits a sequence of SSBs (known as an SSB burst) that sweep a set of TX directions [32], as illustrated in the right panel of Fig. 1. Let $T_{\mathrm{SSB}}$ denote the duration of each SSB, $T_{\mathrm{SS}}$ the SSB burst period, and $N_{\mathrm{SSB}}$ the number of different TX directions swept in each SSB burst period ${ }^{4}$, which depends on the TX codebook as shown in the right panel of Fig. 1. To save power, we assume in Section III that in each SSB burst period, the UE chooses to track $K \leq N_{\mathrm{SSB}} \mathrm{SSB}$ time slots (see right panel of Fig. 1, where $K=4$ and $N_{\mathrm{SSB}}=8$ ). During other SSB time slots, the UE can go to sleep and save power by switching off its RFFE.

Network Model with Carrier Aggregation: Resilience to blockage at mmWave frequencies necessitates macro-diversity, i.e., the UE must be connected to multiple cells [22], [33]. To this end, we assume that the UE is connected to all $N_{\text {cell }}$ gNBs via carrier aggregation, a key feature in 3GPP systems that enables simultaneous connections to multiple cells [34]. The cells may operate in either different component carriers or within the same component carrier ${ }^{5}-$ the analysis for this paper is identical. We also assume that the cells are synchronized so that the SSB time slots from different cells are aligned.

\section{B. Power Consumption in Connected Mode DRX}

The UE power consumption in connected mode DRX depends on the choice of beamforming architecture (analog or digital). At a carrier frequency $f_{c}$ with beamforming scheme BF $\in$ \{Analog, Digital\}, a UE in connected mode DRX in the pre-data arrival phase will need to periodically wake up for the following three events:

- Monitoring SSBs to measure the channel quality (beam measurements), which consumes $P_{\mathrm{BM}}^{f_{c}, \mathrm{BF}}$ units of power per SSB.

- Listening to assignments, which consumes $P_{\mathrm{LS}}^{f_{c}, \mathrm{BF}}$ units of power

- Beam reporting, which consumes $P_{\mathrm{BR}}^{f_{c}, \mathrm{BF}}$ units of power.

Among these, $P_{\mathrm{BM}}^{f_{c}, \mathrm{BF}}$ scales with the number of links that are tracked, while the other procedures have a fixed power cost. Hence, if the UE tracks a large number of links, $P_{\mathrm{BM}}^{f_{c}, \mathrm{BF}}$ could potentially

\footnotetext{
${ }^{4}$ In general, it is not necessary for the gNB to sweep through all the TX directions in its codebook in every SSB burst period, and thus, $N_{\mathrm{SSB}} \leq N_{\mathrm{TX}}$

${ }^{5}$ According to [35], the UE can track up to 21 inter and intra-carrier frequency cells.
} 


\begin{tabular}{|c|c|c|c|c|c|c|}
\hline \multirow[t]{2}{*}{ Task } & \multicolumn{2}{|c|}{ Fraction of time awake } & \multicolumn{2}{|c|}{$\begin{array}{l}\text { Power Consumption } \\
\text { Analog beamforming } \\
(\mathrm{mW})\end{array}$} & \multicolumn{2}{|c|}{$\begin{array}{l}\text { Power Consumption } \\
\text { Digital beamforming } \\
(\mathrm{mW})\end{array}$} \\
\hline & $28 \mathrm{GHz}$ & $140 \mathrm{GHz}$ & $28 \mathrm{GHz}$ & $140 \mathrm{GHz}$ & $28 \mathrm{GHz}$ & $140 \mathrm{GHz}$ \\
\hline $\begin{array}{l}\text { Beam } \\
\text { measurement, } 1 \\
\operatorname{SSB}\left(P_{\mathrm{BM}}^{f_{c}, \mathrm{BF}}\right)\end{array}$ & 0.0017 & 0.00089 & 0.39 & 6.05 & 0.17 & 6.12 \\
\hline $\begin{array}{l}\text { Beam } \\
\text { measurement, all } \\
\text { SSBs } \\
\left(N_{\mathrm{SSB}} \cdot P_{\mathrm{BM}}^{f_{c}, \mathrm{BF}}\right)\end{array}$ & 0.114 & 0.0571 & 24.96 & 774.4 & 10.88 & 783.36 \\
\hline $\begin{array}{l}\text { Listening for } \\
\text { assignments } \\
\left(P_{\mathrm{LS}}^{f_{c}, \mathrm{BF}}\right)\end{array}$ & 0.00625 & 0.00625 & 1.35 & 42.35 & 0.61 & 42.86 \\
\hline $\begin{array}{l}\text { Beam reporting } \\
\text { on } \mathrm{PUCCH} \\
\left(P_{\mathrm{BR}}^{f_{c}, \mathrm{BF}}\right)\end{array}$ & 0.00357 & 0.00178 & 0.77 & 12.06 & 0.34 & 12.21 \\
\hline
\end{tabular}

TABLE I: Estimated awake time and power consumption in connected mode DRX for the parameters defined in Table IX in the appendix.

become the dominant source of power consumption. The total power consumed by the UE RFFE, denoted by $P_{\mathrm{RX}}^{f_{c}, \mathrm{BF}}$, is given by:

$$
P_{\mathrm{RX}}^{f_{c}, \mathrm{BF}}=P_{\mathrm{BM}}^{f_{c}, \mathrm{BF}}+P_{\mathrm{BR}}^{f_{c}, \mathrm{BF}}+P_{\mathrm{LS}}^{f_{c}, \mathrm{BF}}
$$

In Table I, we present estimates of $P_{\mathrm{BM}}^{f_{c}, \mathrm{BF}}, P_{\mathrm{BR}}^{f_{c}, \mathrm{BF}}$ and $P_{\mathrm{LS}}^{f_{c}, \mathrm{BF}}$, based on [10]. For further details, we refer the reader to the appendix. Based on Table I, we make the following remarks:

Remark 1 (Digital beamforming with low-resolution ADCs). Conventionally, digital beamforming is believed to be more power hungry than analog beamforming due to the presence of multiple RF chains. However, a major source of the increased power consumption is the high resolution of the ADCs [36]. Recent works [5], [10] have shown that digital beamforming with low-resolution ADCs can significantly lower the RFFE power consumption with virtually no performance degradation. Throughout this paper, we assume digital beamforming with lowresolution (4-bit) ADCs. 
Remark 2 (Beam tracking and connected mode DRX). Beam measurement on all the SSBS (i.e., tracking the maximum permitted number of links) is by far the largest source of power consumption in connected mode DRX, regardless of carrier frequency and the beamforming architecture.

Remark 3 (Power consumption at $140 \mathrm{GHz}$ ). Digital beamforming at $28 \mathrm{GHz}$ is more power efficient than analog beamforming at $28 \mathrm{GHz}$, while both beamforming architectures have similar power consumption at $140 \mathrm{GHz}$. Relative to $28 \mathrm{GHz}$, the total power consumption at $140 \mathrm{GHz}$ increases by a factor of 70 (35) for digital (analog) beamforming; thus, saving power at $140 \mathrm{GHz}$ is crucial during link tracking.

Remarks 2 and 3 motivate the need for the UE to reduce the number of links to track in connected mode DRX in order to save power and thereby, preserve the effectiveness of DRX as a power saving mechanism. Thus, in the next section, we restrict the number of links that can be tracked at any time and then represent the choice of links to track over time as the outcome of a feasible policy for a MP-MAB problem.

\section{PROBlem Formulation}

We index the SSB burst periods by $t=0,1, \ldots$, and let $\gamma_{i l k}(t)$ denote the measured channel quality (i.e., SNR) from cell $i=1, \ldots N_{\text {cell }}$, in TX direction $l=1, \ldots N_{\mathrm{TX}}$, and $\mathrm{RX}$ direction $k=1 \ldots N_{\mathrm{RX}}$. The tensor $\gamma_{i l k}$ depends on the UE motion, blocking, small-scale fading, and other channel characteristics. Henceforth, we refer to the triplet $(i, l, k)$ as a link.

Let $\mathcal{A}(t)$, which we refer to as the tracking set, denote the set of links over which the UE chooses to measure the channel quality $\gamma_{i l k}(t)$ in the $t$-th SB burst period. In general, $\mathcal{A}(t)$ can be viewed as the outcome of a policy, $\Pi($.$) , in the following manner:$

$$
\mathcal{A}(t)=\Pi\left(\left\{\gamma_{i l k}(s) \in \mathcal{A}(s), s<t\right\}\right),
$$

where, at each SSB burst period $t$, the choice of links to track is a function of past decisions. The links in $\mathcal{A}(t)$ depend on: (a) the SSB time slots the UE is awake for in the $t$-th SSB burst period (which fixes the TX directions at the gNBs), and (b) the RX directions along which the UE measures, which is a function of the beamforming architecture. For instance, with a single $\mathrm{RF}$ chain and analog beamforming, the UE can only the measure channel quality along a single 
RX direction in each SSB time slot (i.e., TX direction) that it is awake for in the $t$-th SSB burst period. Thus, with carrier aggregation, the UE can track each of the $N_{\text {cell }}$ links corresponding to the TX-RX direction pair associated with an awake SSB time slot. On the other hand, with $N_{\mathrm{RX}} \mathrm{RF}$ chains and fully digital beamforming, the UE can measure the channel quality along all $N_{\mathrm{RX}} \mathrm{RX}$ directions in each SSB time slot that it is awake for in the $t$-th SSB burst period. Therefore, with carrier aggregation, the UE can track each of the $N_{\text {cell }} N_{\mathrm{RX}}$ links associated with an awake SSB time slot.

To reduce power consumption, we limit the number of awake SSB time slots in an SSB burst period to $K\left(1 \leq K \leq N_{\mathrm{SSB}}\right)$. Thus, the number of links that a UE can track in each SSB burst period, which is denoted by $L$, depends on $K$ in the following manner:

$$
L:=|\mathcal{A}(t)|= \begin{cases}K N_{\text {cell }}, & \text { for Analog beamforming } \\ K N_{\text {cell }} N_{\mathrm{RX}}, & \text { for Digital beamforming }\end{cases}
$$

Eqn. (3) captures the trade-off between power consumption and performance: the size of the tracking set $(L)$ increases if the UE is awake for longer (i.e., as $K$ increases), which increases both the power consumption as well as the UE's probability of tracking the link with the highest SNR.

Remark 4 (Maximum number of links a UE is permitted to track). Let $L_{\max }$ denote the maximum value of $L$, corresponding to $K=N_{\mathrm{SSB}}$ in (3). $L_{\max }$ is the maximum number of links that a $U E$ is permitted to track in each SSB burst period, which is distinct from the number of available links $\left(N_{\text {cell }} N_{\mathrm{TX}} N_{\mathrm{RX}}\right)$. In general, $L_{\max } \leq N_{\text {cell }} N_{\mathrm{TX}} N_{\mathrm{RX}}$, since it is not necessary for the gNBs to sweep through all $N_{\mathrm{TX}}$ TX directions in each SSB burst period (i.e., $N_{\mathrm{SSB}} \leq N_{\mathrm{TX}}$ ). In addition, $L_{\max }$ reduces by a factor of $N_{\mathrm{RX}}$ for analog beamforming, since the UE is constrained to track links along a single $R X$ direction in each SSB burst period.

Given $K$, we choose $\mathcal{A}(t)$ to maximize the measured SNR. Intuitively, this involves tracking the $L$ strongest links over time, which can be modeled as a multiple-play multi-armed bandit (MP-MAB) problem. In our context, the arms correspond to links and at $t$, a policy $\Pi$ choose $L$ links (multiple plays) out of a total of $N_{\text {cell }} N_{\mathrm{TX}} N_{\mathrm{RX}}$. Let $\gamma_{\max }^{\Pi}(t)$ denote the reward of $\Pi$, which is given by

$$
\gamma_{\max }^{\Pi}(t):=\max _{(i, l, k) \in \mathcal{A}(t)} \gamma_{i l k}(t)
$$


The aim of policy is to minimize the rate of growth of the cumulative regret, $R^{\Pi}(t)$, which is given by:

$$
R^{\Pi}(T):=\mathbb{E}\left[\sum_{t=1}^{T} \max _{(i, l, k)} \gamma_{i l k}(t)\right]-\mathbb{E}\left[\sum_{t=1}^{T} \gamma_{\max }^{\Pi}(t)\right] .
$$

In choosing $\mathcal{A}(t)$, a policy needs to trade-off between two competing requirements: exploration and exploitation. The latter, where the UE tracks the $L$ links with the highest (measured) average SNR, helps to minimize the rate of growth of $R^{\Pi}($.$) in (5). However, the SNR statistics of the$ links can change with time; hence, the UE needs to track sub-optimal links from time to time (i.e, exploration) to adapt to any changes to the set of the strongest $L$ links.

From (5), we can see that formulating a policy with provable performance guarantees (e.g. sub-linear growth of $R^{\Pi}(T)$ ) for mmWave and sub-THz systems depends on knowing how the joint statistics of the link SNRs (i.e., $\left.\left\{\gamma_{i l k}(t), \forall i, l, k\right\}\right)$ evolve with time. However, in a multipath environment with mobility, the channel dynamics of a collection of directional links is hard to characterize due to the intermittent nature of links, as well as correlation across links. Hence, in order to provide useful insights for mmWave and sub-THz systems, such as (a) the fraction of links (i.e., $K / N_{\mathrm{SSB}}$ ) that need to be tracked for at most $1 \%$ outage probability, and (b) the throughput degradation (in case of data arrival) due to tracking only a limited number of links, we consider four policies and evaluate their performance in terms of these criteria using detailed system-level simulations at $28 \mathrm{GHz}$ and $140 \mathrm{GHz}$, representative of a 5G mmWave and a hypothetical $6 \mathrm{G}$ sub-THz operating environment, respectively. The policies are (i) the $\epsilon$-greedy algorithm [37], (ii) Thompson Sampling [38], (iii) the Upper confidence bound (UCB) algorithm [39], and (iv) the $\left(L-L_{m}\right)$ round-robin policy. The first three are well-known policies for solving MAB problems, but typically assume stationary rewards (i.e., stationary SNR distribution over each link) and independence across arms (links). Hence, these policies are sub-optimal in our case. The fourth policy is a heuristic adapted from our earlier work [1].

Despite their sub-optimality, comparing the performance of multiple policies and identifying a winner among them based on their relative performance enables us to draw (mild) achievability conclusions like "a well-designed link tracking policy should simultaneously realize $75 \%$ power savings, $95 \%$ of the maximum throughput, and $<1 \%$ outage probability in a $5 \mathrm{G}$ mmWave environment at $28 \mathrm{GHz}$ with digital beamforming at the UE", because the winning policy meets these targets in our realistic system-level simulations. From an engineering perspective, 
we believe that conclusions like these are valuable, despite lacking in rigor, and are the key results of this paper. In contrast, we stress that conclusions like "the $\epsilon$-greedy policy performs best at $28 \mathrm{GHz}$ with analog beamforming at the UE', because it happens to be the winning policy in our simulations, are of little significance value and we refrain from making them.

We review the policies in the next section. Due to their sub-optimality, we do not delve into a detailed mathematical treatment of the policies; for these, we direct the reader to the references provided. However, we attempt to provide enough intuition behind their working, specifically on their exploration and exploitation mechanisms.

\section{Link Tracking Policies for Connected Mode DRX}

Before reviewing the policies, we first define some common quantities. Let $\mathcal{G}_{n}(t):=\left\{\gamma_{n}(s)\right.$ : $n \in \mathcal{A}[s], s \leq t\}$ denote the set of SNR values measured for the $n$-th link $\left(n=1, \cdots, N_{\text {cell }} N_{\mathrm{TX}} N_{\mathrm{RX}}\right)$

up to the $t$-th SSB burst period. Hence, $\hat{N}_{n}(t):=\left|\mathcal{G}_{n}(t)\right|$ denotes the number of times the $n$-th link has been included in the tracking set up to the $t$-th SSB burst period. Finally, let $\bar{\gamma}_{n}(t)$ denote the mean measured SNR of the $n$-th link up to the $t$-th SSB burst period (i.e., the sample mean of the elements of $\left.\mathcal{G}_{n}(t)\right)$.

\section{A. Upper Confidence Bound (UCB) Algorithm}

We adapt the policy presented in [39] for multiple play MAB problems. The UCB link tracking policy is detailed in Algorithm 1. In the initialization phase, the policy measures the SNR of every link once, before deciding which links to track over time. During the operation stage, (6) determines the SSB burst periods when exploration and exploitation take place. After convergence, the first term in (6) helps in continuing to track links with high average SNR (large $\bar{\gamma}_{n}(t)$ ), which constitutes exploitation. However, as $t$ increases in (6), the second term eventually becomes large enough for an infrequently tracked (weak) link (i.e., small $\hat{N}_{n}(t)$ ) to be included in the tracking set, which constitutes exploration.

Remark 5. A drawback of the UCB policy is the requirement to measure all the links initially in our simulation setup in Section $V$, for $K=1$ (corresponding to the least power consumption) at $28 \mathrm{GHz}$ with analog beamforming at the UE, the initialization takes $10.2 \mathrm{~s}$ to finish, which 
is orders of magnitude greater than the channel coherence time (around $21 \mathrm{~ms})^{6}$. Hence, the channel statistics would have changed considerably by the time the UE decides to track (exploit) the best links found during the initial exploration. However, as $K$ increases, the initialization time reduces by a factor of $K$ and the policy performs better, as seen in Fig. $9 a$ (UCB curve) later in Section VI-B.

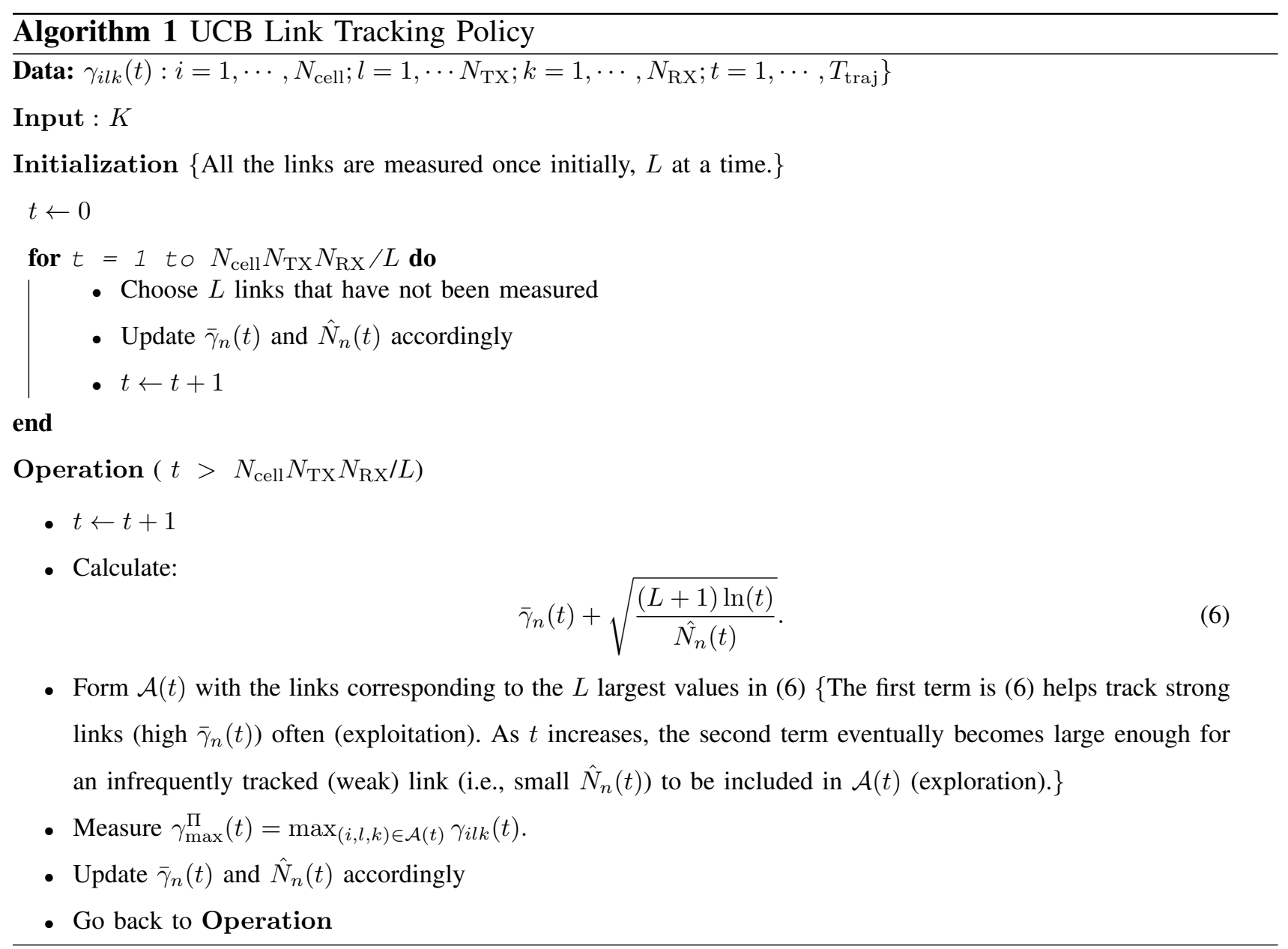

\section{B. $\epsilon$-Greedy Algorithm}

The $\epsilon$-greedy link tracking policy is detailed in Algorithm 2. Unlike the UCB policy, it uses only the average link SNRs, $\bar{\gamma}_{n}(t)$, to determine the tracking set. The exploration and exploitation

${ }^{6}$ For $K=1$ with analog beamforming i.e. the most power efficient case, the UE can measure only $N_{\text {cell }}$ links (due to carrier aggregation) in one SSB burst period, out of a total of $N_{\text {cell }} N_{\mathrm{TX}} N_{\mathrm{RX}}$. Hence, the time taken for the initialization phase of Algorithm 5 equals $N_{\mathrm{TX}} N_{\mathrm{RX}} T_{\mathrm{SS}}=10.2 \mathrm{~s}$ at $28 \mathrm{GHz}$, for values chosen from Table IX. For a UE with an average velocity, $v$, of $0.5 \mathrm{~m} / \mathrm{s}$ (human), the coherence time, $T_{\text {coh }} \approx c /\left(f_{c} v\right) \approx 21 \mathrm{~ms}[40]$. 


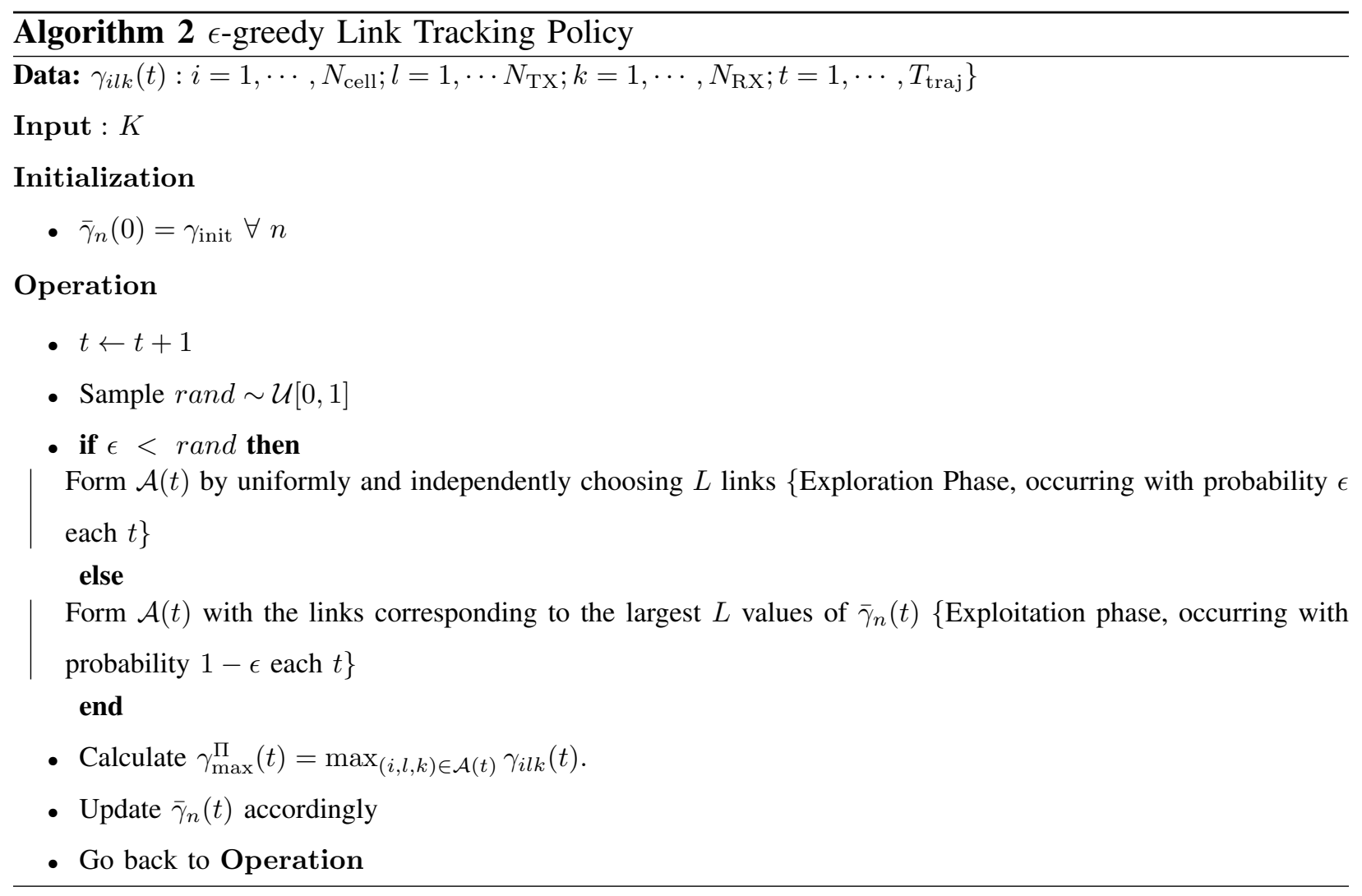

phases of this policy is determined by a parameter $\epsilon \in(0,1)$. At each $t$, the policy independently explores with probability $\epsilon$ and exploits with probability $1-\epsilon$. During exploration, the policy uniformly and independently chooses $L$ links to track, while in the exploitation phase, it tracks the $L$ links with the highest $\bar{\gamma}_{n}(t)$. Initially, $\bar{\gamma}_{n}(0)=\gamma_{\text {init }} \forall n$, a suitably large $\mathrm{SNR}^{7}$ that ensures that the policy tracks each link at least once before converging. The choice of $\epsilon$ is discussed in Section VI.

\section{Thompson Sampling}

In contrast to the previous policies, Thompson sampling adopts a Bayesian approach, where the average SNR of the $n$-th link is assumed to be a random variable, $X_{n}$. Assuming independence across links ${ }^{8}$, let $f\left(X_{n} \mid \mathcal{G}_{n}(t)\right)$ denote the posterior probability density function (pdf) of $X_{n}$, given the measured values of $\gamma_{n}$ up to the $t$-th SSB burst period. Starting from a prior pdf $\zeta_{n}$,

\footnotetext{
${ }^{7}$ We assume $\gamma_{\text {init }}=19.6 \mathrm{~dB}$, which corresponds to the minimum SNR needed to decode the highest modulation and coding scheme (MCS) level in 3GPP, according to (26).

${ }^{8}$ The SNRs of two or more links are, in general, not independent, due to correlated blocking.
} 
$f\left(X_{n} \mid \mathcal{G}_{n}(t)\right)$ is updated every time the $n$-th link is included in the tracking set (based on $\bar{\gamma}_{n}(t)$ and $\hat{N}_{n}(t)$ ), using Bayes' rule. Thus, the policy aims to track the links with the $L$ largest values of the posterior mean $\mathbb{E}\left[X_{n} \mid \mathcal{G}_{n}(t)\right]$.

The Thompson sampling based link tracking policy, adapted from [38] ${ }^{9}$, is presented in Algorithm 3. The performance of this policy depends on the choice of $\zeta_{n}$ and the resulting $f\left(X_{n} \mid \mathcal{G}_{n}(t)\right)$. To evaluate the latter in a tractable manner, conjugate priors ${ }^{10}$ are commonly assumed for $\zeta_{n}$. The choice of distributions is discussed in Section VI-A.

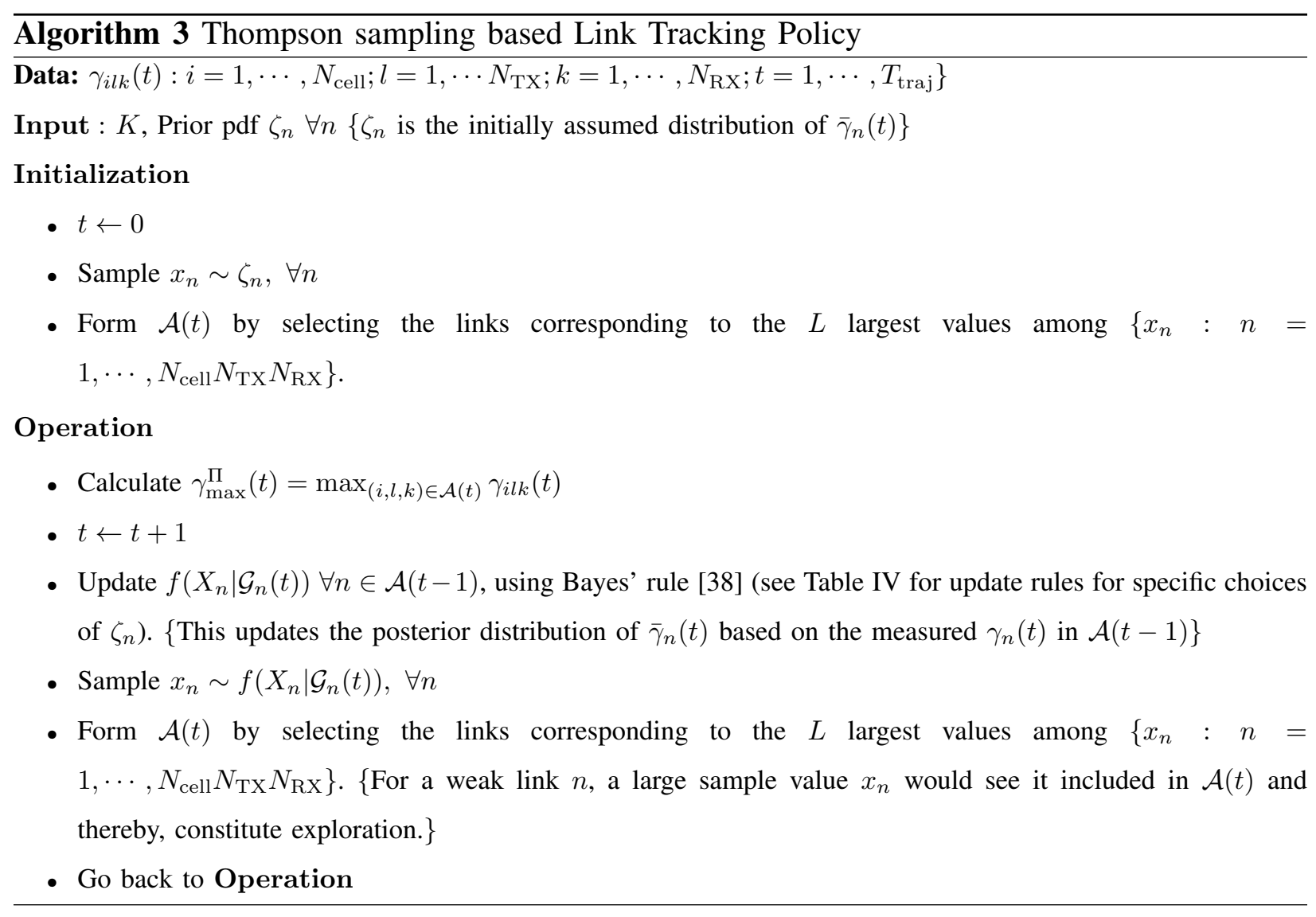

\section{D. $\left(L-L_{m}\right)$ Round Robin Policy}

Finally, we consider a policy with one-step memory (i.e., $s=t-1$ in (2)), which is a modified version of the policy presented in [1]. For the current SSB burst period $t$, the policy

${ }^{9}$ To the best of our knowledge, there exists no formal Thompson sampling policy for MP-MAB problems; hence, we adapt the conventional single play Thompson sampling policy for multiple plays.

${ }^{10}$ For the likelihood function, $f\left(\mathcal{G}_{n} \mid X_{n}\right), \zeta_{n}$ is a conjugate prior if the posterior pdf, $f\left(X_{n} \mid \mathcal{G}_{n}\right)$, belongs to same family of distributions as $\zeta_{n}$. 
retains the $L_{m}\left(1 \leq L_{m}<L\right)$ strongest links from $\mathcal{A}(t-1)$ based on the instantaneous SNR $\gamma_{n}(t)$ (exploitation) and selects the other $L-L_{m}$ links independently and uniformly from the remaining links (exploration). We refer to this as $\left(L-L_{m}\right)$ round robin policy, which is summarized in Algorithm 4. The choice of $L_{m}$ is discussed in Section VI-A.

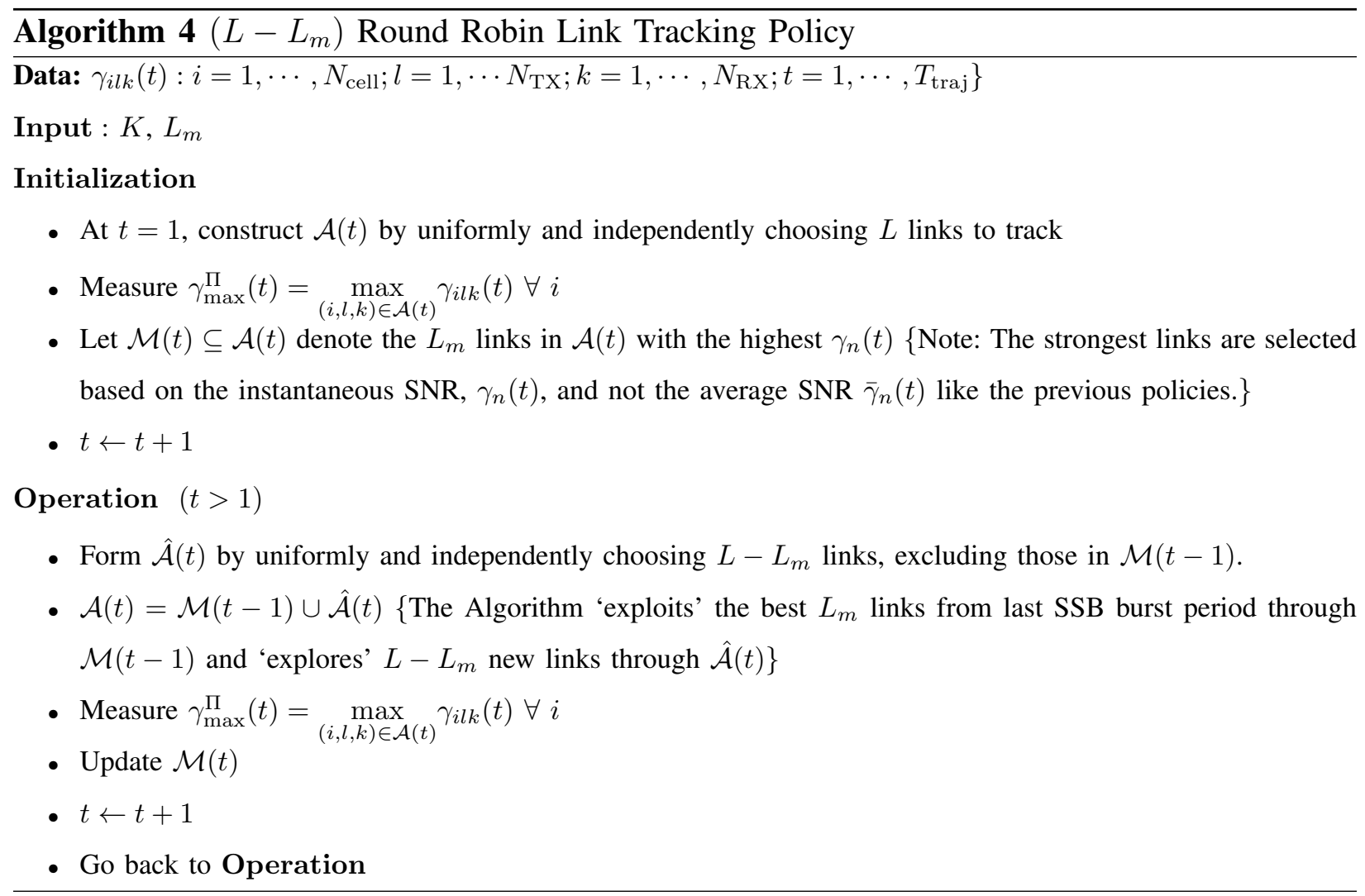

\section{Simulation Setup}

Comprehensive link-level simulations are used to generate the channel trajectories at $28 \mathrm{GHz}$ and $140 \mathrm{GHz}$. Nine gNBs are deployed $\left(N_{\text {cell }}=9\right)$ in a $400 \mathrm{~m} \times 400 \mathrm{~m}$ and the cell radius, $r$, of each gNB is $100 \mathrm{~m}$. The heights of the gNBs and the UE are set to $10 \mathrm{~m}$ and $1.7 \mathrm{~m}$, respectively, in accordance with the 3GPP 'UMi' (urban microcell) specification [41]. At the start of each channel trajectory, the UE is dropped according to a two-dimensional uniform distribution that covers the grid. 


\section{A. Array Sizes and Beamforming Codebook}

In accordance with Table IX, a $4 \times 2(8 \times 8)$ uniform planar array (UPA) with $\lambda / 2$ antenna spacing is assumed at the UE and an $8 \times 8(16 \times 16) \mathrm{UPA}$ at the $\mathrm{gNB}$ for $f_{c}=28(140) \mathrm{GHz}$. We consider a simple beamforming codebook, where the main lobes of the antenna patterns associated with the beamforming vectors span the hemisphere, equally spaced in both azimuth and elevation. Hence, we assume two similar antenna arrays at the UE and gNB for full 360 degree coverage like practical devices [42] (i.e., one array covering the front hemisphere and the other the rear).

1) UE codebook: For a $N_{x}^{\mathrm{RX}} \times N_{y}^{\mathrm{RX}} \mathrm{UPA}$ at the UE (where $\left.N_{\mathrm{RX}}=N_{x}^{\mathrm{RX}} N_{y}^{\mathrm{RX}}\right)$, let $\mathbf{u}\left(\Omega_{\mathrm{UE}}\right) \in$ $\mathbb{C}^{N_{\mathrm{RX}}}$ denote the steering vector along $\Omega_{\mathrm{UE}}:=\left(\phi_{\mathrm{UE}}, \varphi_{\mathrm{UE}}\right)$, where $\phi_{\mathrm{UE}} \in[-\pi, \pi)$ and $\varphi_{\mathrm{UE}} \in$ $[-\pi / 2, \pi / 2]$ denote the orientations along the azimuth and elevation, respectively, from the UE's perspective. The expression for $\mathbf{u}\left(\Omega_{\mathrm{UE}}\right)$, for $\lambda / 2$ antenna spacing, is given by:

$$
\begin{aligned}
\mathbf{u}\left(\Omega_{\mathrm{UE}}\right) & =\left[\left(\mathbf{u}_{1}\left(\Omega_{\mathrm{UE}}\right)\right)^{T} \ldots\left(\mathbf{u}_{N_{x}^{\mathrm{RX}}}\left(\Omega_{\mathrm{UE}}\right)\right)^{T}\right] \\
\text { where } \mathbf{u}_{m_{r}}\left(\Omega_{\mathrm{UE}}\right) & :=\left[\begin{array}{c}
\exp \left(j \pi\left(m_{r} \sin \varphi_{\mathrm{UE}} \cos \phi_{\mathrm{UE}}+\sin \varphi_{\mathrm{UE}} \sin \phi_{\mathrm{UE}}\right)\right) \\
\vdots \\
\exp \left(j \pi\left(m_{r} \sin \varphi_{\mathrm{UE}} \cos \phi_{\mathrm{UE}}+N_{y}^{\mathrm{RX}} \sin \varphi_{\mathrm{UE}} \sin \phi_{\mathrm{UE}}\right)\right)
\end{array}\right] \in \mathbb{C}^{N_{y}^{\mathrm{RX}}},
\end{aligned}
$$

and $m_{r}=1, \cdots, N_{x}^{\mathrm{RX}}$.

Let $\Omega_{k}^{(1)}:=\left(\phi_{k}^{(1)}, \varphi_{k}^{(1)}\right)$ and $\Omega_{k}^{(2)}:=\left(\phi_{k}^{(2)}, \varphi_{k}^{(2)}\right)$ denote the main lobe orientations along the $k$-th $\mathrm{RX}$ direction $\left(k=1, \cdots, N_{\mathrm{RX}}\right)$ at the $\mathrm{UE}$ for the front and rear antenna arrays, respectively. The expressions for the azimuth and elevation angles are given by

$$
\begin{aligned}
& \phi_{k}^{(1)}=\left(\frac{\left((k-1) \bmod N_{x}^{\mathrm{RX}}\right)+1}{N_{x}^{\mathrm{RX}}}\right) \pi+\frac{\pi}{2} \\
& \phi_{k}^{(2)}=\left(\frac{\left((k-1) \bmod N_{x}^{\mathrm{RX}}\right)+1}{N_{x}^{\mathrm{RX}}}\right) \pi-\frac{\pi}{2} \\
& \varphi_{k}^{(1)}=\varphi_{k}^{(2)}=\left(\frac{\left\lfloor(k-1) / N_{x}^{\mathrm{RX}}\right\rfloor+1}{N_{y}^{\mathrm{RX}}}\right) \pi-\frac{\pi}{2}
\end{aligned}
$$

Let $\mathcal{W}_{k}:=\left\{\mathbf{w}_{k}^{(1)}, \mathbf{w}_{k}^{(2)}\right\}$ denote the set of UE beamforming vectors corresponding to the $k$-th $\mathrm{RX}$ direction, where $\mathbf{w}_{k}^{(1)}, \mathbf{w}_{k}^{(2)} \in \mathbb{C}^{N_{\mathrm{Rx}}}$ correspond to the front and rear antenna arrays, respectively. 
Their expressions are given by

$$
\begin{aligned}
& \mathbf{w}_{k}^{(1)}=\frac{1}{\sqrt{N_{\mathrm{RX}}}} \mathbf{u}\left(\Omega_{k}^{(1)}\right) \\
& \mathbf{w}_{k}^{(2)}=\frac{1}{\sqrt{N_{\mathrm{RX}}}} \mathbf{u}\left(\Omega_{k}^{(2)}\right),
\end{aligned}
$$

where $\mathbf{u}\left(\Omega_{k}^{(1)}\right)$ and $\mathbf{u}\left(\Omega_{k}^{(2)}\right)$ are obtained from (7), (8), (9) and (10).

2) gNB codebook: Similarly, for a $N_{x}^{\mathrm{TX}} \times N_{y}^{\mathrm{TX}} \mathrm{UPA}$ at the gNB (where $N_{\mathrm{TX}}=N_{x}^{\mathrm{TX}} N_{y}^{\mathrm{TX}}$ ), let $\mathbf{v}\left(\Omega_{\mathrm{gNB}}\right) \in \mathbb{C}^{N_{\mathrm{TX}}}$ denote the steering vector along $\Omega_{\mathrm{gNB}}:=\left(\phi_{\mathrm{gNB}}, \varphi_{\mathrm{gNB}}\right)$, where $\phi_{\mathrm{gNB}} \in[-\pi, \pi)$ and $\varphi_{\mathrm{gNB}} \in[-\pi / 2, \pi / 2]$ are the orientations along the azimuth and elevation, respectively, from the gNB's perspective. $\mathbf{v}\left(\Omega_{\mathrm{gNB}}\right)$, for $\lambda / 2$ antenna spacing is given by:

$$
\begin{aligned}
\mathbf{v}\left(\Omega_{\mathrm{gNB}}\right) & =\left[\left(\mathbf{v}_{1}\left(\Omega_{\mathrm{gNB}}\right)\right)^{T} \ldots\left(\mathbf{v}_{N_{x}^{\mathrm{TX}}}\left(\Omega_{\mathrm{gNB}}\right)\right)^{T}\right] \\
\text { where } \mathbf{v}_{m_{t}}\left(\Omega_{\mathrm{gNB}}\right) & :=\left[\begin{array}{c}
\exp \left(j \pi\left(m_{t} \sin \varphi_{\mathrm{gNB}} \cos \phi_{\mathrm{gNB}}+\sin \varphi_{\mathrm{gNB}} \sin \phi_{\mathrm{gNB}}\right)\right) \\
\vdots \\
\exp \left(j \pi\left(m_{t} \sin \varphi_{\mathrm{gNB}} \cos \phi_{\mathrm{UE}}+N_{y}^{\mathrm{TX}} \sin \varphi_{\mathrm{gNB}} \sin \phi_{\mathrm{gNB}}\right)\right)
\end{array}\right] \in \mathbb{C}^{N_{y}^{\mathrm{TX}},}
\end{aligned}
$$

and $m_{t}=1, \cdots, N_{x}^{\mathrm{TX}}$.

Let $\Omega_{l}^{(1)}:=\left(\phi_{l}^{(1)}, \varphi_{l}^{(1)}\right)$ and $\Omega_{l}^{(2)}:=\left(\phi_{l}^{(2)}, \varphi_{l}^{(2)}\right)$ denote the main lobe orientations along the $l$-th TX direction $\left(l=1, \cdots, N_{\mathrm{TX}}\right)$ at the $\mathrm{gNB}$ for the front and rear antenna arrays, respectively. The expressions for the azimuth and elevation angles are given by

$$
\begin{aligned}
& \phi_{l}^{(1)}=\left(\frac{\left((l-1) \bmod N_{x}^{\mathrm{TX}}\right)+1}{N_{x}^{\mathrm{TX}}}\right) \pi+\frac{\pi}{2} \\
& \phi_{l}^{(2)}=\left(\frac{\left((l-1) \bmod N_{x}^{\mathrm{TX}}\right)+1}{N_{x}^{\mathrm{TX}}}\right) \pi-\frac{\pi}{2} \\
& \varphi_{l}^{(1)}=\varphi_{l}^{(2)}=\left(\frac{\left\lfloor(l-1) / N_{x}^{\mathrm{TX}}\right\rfloor+1}{N_{y}^{\mathrm{TX}}}\right) \pi-\frac{\pi}{2}
\end{aligned}
$$

Let $\mathcal{F}_{l}:=\left\{\mathbf{f}_{l}^{(1)}, \mathbf{f}_{l}^{(2)}\right\}$ denote the collection of gNB beamforming vectors corresponding to the l-th $\mathrm{RX}$ direction, where $\mathbf{f}_{l}^{(1)}, \mathbf{f}_{l}^{(2)} \in \mathbb{C}^{N_{\mathrm{TX}}}$, correspond to the front and rear antenna arrays, respectively. Their expressions are given by

$$
\begin{aligned}
& \mathbf{f}_{l}^{(1)}=\frac{1}{\sqrt{N_{\mathrm{TX}}}} \mathbf{v}\left(\Omega_{l}^{(1)}\right) \\
& \mathbf{f}_{l}^{(2)}=\frac{1}{\sqrt{N_{\mathrm{TX}}}} \mathbf{v}\left(\Omega_{l}^{(2)}\right),
\end{aligned}
$$

where $\mathbf{v}\left(\Omega_{l}^{(1)}\right)$ and $\mathbf{v}\left(\Omega_{l}^{(2)}\right)$ are obtained from (13), (14), (15) and (16). 


\section{B. Blockage modeling}

Blockers are placed using Poisson point process (PPP) [43] with blocker density $\lambda_{b}=$ $0.01 \mathrm{~m}^{-2}$. The blockers can be human or vehicular with equal probability. The dimensions and velocities of the blockers are chosen according to 3GPP modeling specifications [41] i.e., the height and width for a human (vehicular) blocker are $1.7 \mathrm{~m}(1.5 \mathrm{~m})$ and $0.3 \mathrm{~m}(4.5 \mathrm{~m})$, respectively. The magnitude of the velocities of human (vehicular) blockers are iid uniform between $[0,3]([0,100]) \mathrm{km} / \mathrm{hr}$. The blockers are assumed to move in the $x y$ plane.

We choose the Double Knife Edge Diffraction (DKED) model ${ }^{11}$ for calculating the blockage loss, since to the best of our knowledge, there is no parametric blockage model for $140 \mathrm{GHz}$. On the other hand, the DKED model is physics-based and holds for all frequencies. Moreover, measurement results in [45] show that the DKED model is within a few $\mathrm{dB}$ of the blockage loss at mmWave frequencies. Hence, we believe that the DKED model would provide a reasonably accurate estimate of the blockage loss at $140 \mathrm{GHz}$ as well. Fig. 2 shows the blockage loss due to a moving human blocker at $28 \mathrm{GHz}$ and $140 \mathrm{GHz}$ for a link operating at an SNR of $10 \mathrm{~dB}$ in the unblocked state. We see that at $28 \mathrm{GHz}$, the SNR reduces to $0 \mathrm{~dB}$ during the blockage event, which can still support communications at the modulation and coding scheme (MCS) 3 [46, Table 1], according to (26). However, at $140 \mathrm{GHz}$, the SNR drops to $-6 \mathrm{~dB}$, which causes a link outage as it is below the minimum SNR needed $(-4.5 \mathrm{~dB})$ to communicate at MCS 0 , the lowest MCS level. Hence, blockage induced link outages are expected to be more frequent at sub-THz frequencies. From the perspective of connected mode DRX, this means that the UE may be required to track more links to ensure the same level of performance (e.g., outage probability) which highlights the importance of power-efficient link tracking policies at these frequencies.

\section{Non line-of-sight paths}

Stationary reflectors with density $\lambda_{r}=0.01 \mathrm{~m}^{-2}$ are also deployed according to a PPP. We assume that the reflectors do not cause blockage. We form clusters of reflectors based on their proximity [47] and select up to $N_{\text {Cluster }}$ clusters in increasing order of the gNB $\rightarrow$ cluster $\rightarrow$ UE

\footnotetext{
${ }^{11}$ DKED is also known as Blockage Model B in 3GPP specifications [41] and is used in the METIS project [44].
} 

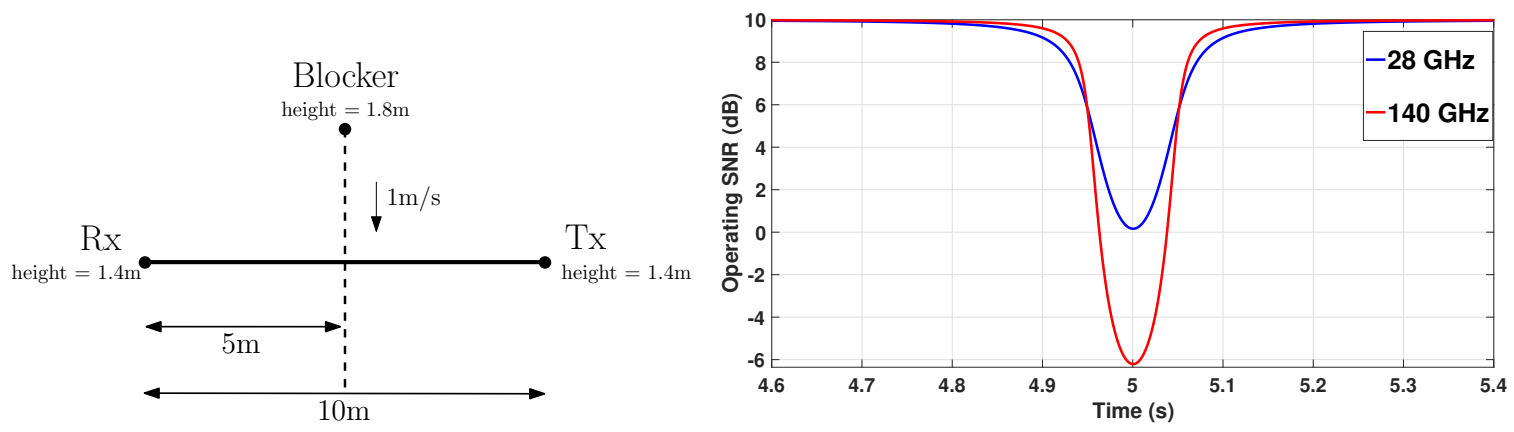

Fig. 2: Illustration of the severity of blockages at mmWave and sub-THz frequencies. Left panel: A moving human blocker based on 3GPP model parameters [41]. Right Panel: Blockage loss suffered by the link due to double knife-edge diffraction.

path length [48], in addition to the line-of-sight path. The reflection loss suffered by the signal is taken to be $7(10) \mathrm{dB}$ at $28(140) \mathrm{GHz}[49]^{12}$.

\section{Mobility}

The reflectors are assumed to be static. The UE and blockers follow the random waypoint mobility model [50]. Under this model, let $\mathbf{x}_{t} \in \mathbb{R}^{2}$ represent the position of an object during the $t$-th SSB burst period. At the next SSB burst period, the position is updated as follows:

$$
\mathbf{x}_{t+1}=\mathbf{x}_{t}+\dot{\mathbf{x}} T_{\mathrm{SS}}
$$

where $\dot{\mathbf{x}}$ is the velocity of the object. The $x$ and $y$ components of the velocity vector are independently chosen according to the distributions in Table II. The UE and the human blockers have similar mobility characteristics, since their velocities are extracted from the same distribution; vehicular blockers have a different velocity distribution. A destination is associated with every mobile object at the start of each simulation, which is changed when the object in question reaches the destination. The destination of the objects are restricted within the simulation grid.

\section{E. SNR Calculation}

Let $\mathbf{H}_{i}(t) \in \mathbb{C}^{N_{\mathrm{RX}} \times N_{\mathrm{TX}}}$ denote the channel gain matrix between the $i$-th gNB and the UE at the $t$-th SSB burst period, due to $N_{\text {Cluster }}$ multipath components (MPCs). For the $p$-th MPC,

\footnotetext{
${ }^{12}$ In general, the reflection loss depends on the material and the angle of incidence. For the sake of simplicity, we do not consider these effects.
} 
let $\Omega_{i, p}^{A}(t):=\left(\phi_{i, p}^{A}(t), \varphi_{i, p}^{A}(t)\right), \Omega_{i, p}^{D}(t):=\left(\phi_{i, p}^{D}(t), \varphi_{i, p}^{A}(t)\right), \alpha_{i, p}(t), \rho_{i, p}(t)$ and $d_{i, p}(t)$ denote the angle of arrival, angle of departure, blockage loss, reflection loss and path length, respectively. Then, $\mathbf{H}_{i}(t)$ can be expressed as follows:

$$
\begin{aligned}
& \mathbf{H}_{i}(t)= \sum_{p=1}^{N_{\text {cluster }}} \sqrt{\frac{\alpha_{i, p}(t) \rho_{i, p}(t) P_{0} c^{2}}{4 \pi^{2} f_{c}^{2} d_{i, p}(t)^{2}}} \mathbf{u}\left(\Omega_{i, p}^{A}(t)\right) \mathbf{v}^{H}\left(\Omega_{i, p}^{D}(t)\right) \times \\
& \quad \exp \left(-j 2 \pi \frac{f_{c}}{c}\left(d_{i, l}(t)-\left\|\dot{\mathbf{x}}_{\mathrm{UE}}\right\| t \cos \varphi_{i, p}^{A}(t) \cos \phi_{i, p}^{A}(t)\right)\right),
\end{aligned}
$$

where $\mathbf{u}($.$) and \mathbf{v}($.$) are obtained from (7) and (13), respectively, \left\|\dot{x}_{\mathrm{UE}}\right\|$ denotes the UE speed, $f_{c}$ the carrier frequency and $c$ the speed of light. The expression for $\gamma_{i l k}(t)$ is as follows:

$$
\gamma_{i l k}(t)=\max _{\substack{\mathbf{w}_{k} \in \mathcal{W}_{k} \\ \mathbf{f}_{l} \in \mathcal{F}_{l}}} \frac{\left|\mathbf{w}_{k}^{H} \mathbf{H}_{i}(t) \mathbf{f}_{l}\right|^{2}}{k_{\mathrm{B}} B N_{F} T_{0}},
$$

where $k_{\mathrm{B}}$ is Boltzmann's constant, $B$ denotes the system bandwidth, $N_{F}$ the noise figure, and $T_{0}$ the temperature.

We generate a total of 100 channel trajectories. For each trajectory, we simulate over 3000 SSB burst periods, with $T_{\mathrm{SS}}=20 \mathrm{~ms}$, amounting to a runtime of $60 \mathrm{~s}$ per trajectory $\left(T_{\text {traj }}\right)$. The list of parameter values used to generate the simulation data is presented in Table II. An inherent weakness of our simulation-based approach is the fact that the results in the following section depend on the values in Table II, and could change for a different set of parameter values. To mitigate this, we have chosen values that are consistent with the 3GPP standards and/or backed by empirical evidence. While this does not guarantee that the parameter values will remain fixed forever, we believe that it provides a reasonable safeguard against large changes, which in turn, lends weight to the conclusions that we draw from our simulation results.

\section{RESULTS AND DISCUSSION}

At mmWave and sub-THz frequencies, the link SNRs in an urban microcell environment are expected to be non-stationary due to UE mobility and the blockage losses caused by mobile blockers. To get an insight into the timescale of SNR variation, we first simulated a single gNBUE link under a horn antenna configuration at both end points, wherein the UE's starting location was kept the same for all trajectories and its movement confined along the line connecting it to the gNB by a one-dimensional version of the mobility model in (19). This restriction ensures the same link (i.e., the same beam directions at the UE and the gNB) over all trajectories at 


\begin{tabular}{|l|l|l|l|l|c|}
\hline Parameters & $28 \mathrm{GHz}$ & \multicolumn{1}{|c|}{$140 \mathrm{GHz}$} & Parameters & $28 \mathrm{GHz}$ & $140 \mathrm{GHz}$ \\
\hline Temperature, $T_{0}$ & \multicolumn{2}{|c|}{$298 \mathrm{~K}$} & Cell radius, $r$ & $100 \mathrm{~m} \mathrm{[51]}$ \\
\hline Bandwidth, $B(\ddagger)$ & $400 \mathrm{MHz}$ & $1.6 \mathrm{GHz}$ & Blocker density & $0.01 \mathrm{~m}^{-2}$ [52] \\
\hline$N_{\mathrm{TX}}(\ddagger)$ & 64 & 256 & Blocker height $(\dagger)$ & $1.4 \mathrm{~m}$ (Vehicular), $1.7 \mathrm{~m}$ (Human) \\
\hline$N_{\mathrm{RX}}(\ddagger)$ & 8 & 64 & Blocker width $(\dagger)$ & $4.8 \mathrm{~m}$ (Vehicular), 0.3 m (Human) \\
\hline$N_{\text {cell }}$ & \multicolumn{2}{|c|}{$9[35]$} & Blocker speed distribution $(\dagger)$ & $\begin{array}{l}\mathcal{U}[0,100] \mathrm{km} / \mathrm{hr} \text { (Vehicular) } \\
\mathcal{U}[0,3] \mathrm{km} / \mathrm{hr} \text { (Human) }\end{array}$ \\
\hline$N_{\text {Cluster }}$ & \multicolumn{2}{|c|}{$4[48]$} & Transmit Power, $P_{0}$ & $23 \mathrm{dBm}[53]$ \\
\hline UE height $(\dagger)$ & \multicolumn{2}{|c|}{$1.7 \mathrm{~m}$} & Noise Figure, $N_{F}$ & $9 \mathrm{~dB}[54]$ \\
\hline gNB height $(\dagger)$ & \multicolumn{2}{|c|}{$10 \mathrm{~m}$} & $T_{\text {SS }}$ & $20 \mathrm{~ms}[55]$ \\
\hline Reflection Loss $[49]$ & $7 \mathrm{~dB}$ & $10 \mathrm{~dB}$ & Scenario & UMi $(\dagger)$ \\
\hline
\end{tabular}

TABLE II: Simulation parameters used for generating channel trajectories (†: [41], $\ddagger:[10])$.

all times. For this setup, the evolution of the average link SNR in increments of $T_{\mathrm{SS}}$ (i.e., the duration between successive SSB burst periods) is plotted in Fig. 3. The fairly large fluctuations in the average SNR over relatively short time scales confirms the non-stationarity of the operating environment, and reinforces the difficulty of formulating a link tracking policy with provable performance guarantees.

In the pre-data arrival phase, the outage probability is an important performance metric that affects both the throughput and the latency ${ }^{13}$, in case there is data arrival on the downlink. In Sections VI-A and VI-B, we analyze the outage performance of the policies discussed in Section IV, for analog and digital beamforming at the UE, at 28 and $140 \mathrm{GHz}$. The purpose of this analysis is to quantify the amount of power the UE can potentially save, subject to satisfying an outage probability of at most $1 \%$. The outage probability for a policy $\Pi$, denoted by $P_{\text {out }}^{\Pi}$, is defined as follows:

$$
P_{\text {out }}^{\Pi}:=\mathbb{P}\left(\gamma_{\max }^{\Pi}(t)<\gamma_{\text {tgt }}\right),
$$

where $\gamma_{\text {tgt }}$ is the minimum SNR required to communicate using MCS 0. The value of $\gamma_{\text {tgt }}$ can be obtained from (26) for $u=0$.

\footnotetext{
${ }^{13} \mathrm{~A}$ UE in outage needs to trigger initial access procedures to re-establish connectivity to the network, which incurs some delay and adds to the overall latency.
} 


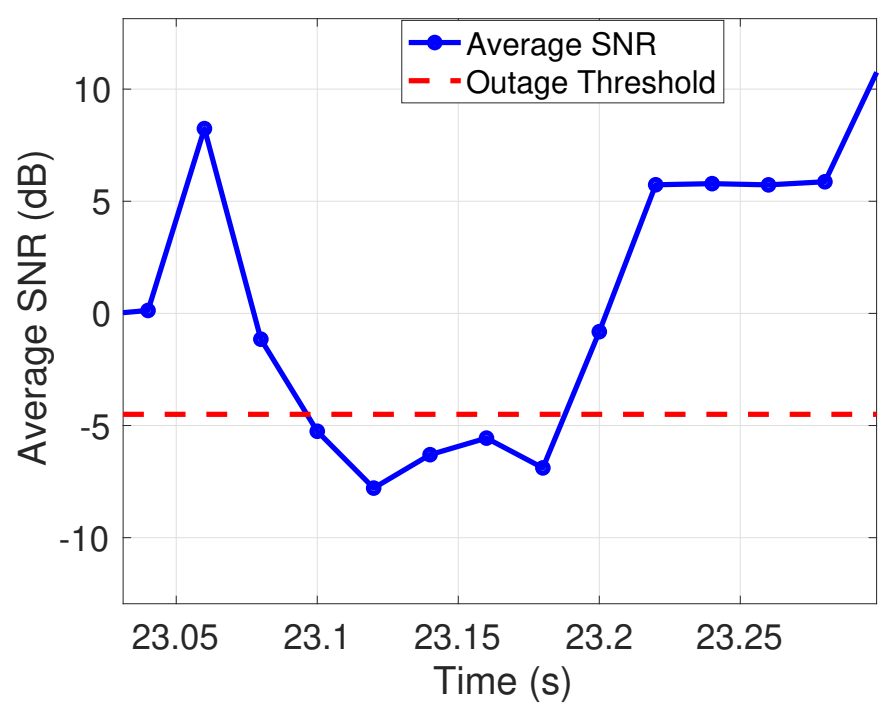

Fig. 3: Evolution of the average link SNR over increments of $T_{\mathrm{SS}}=20 \mathrm{~ms}$ (i.e., the interval between successive SSB burst periods) for a horn antenna configuration at $28 \mathrm{GHz}$. The fairly large fluctuations in the average SNR over the short time scales points to a non-stationary operating environment.

\section{A. Parameter tuning for the $\epsilon$-greedy, Thompson sampling and $\left(L-L_{m}\right)$ Round robin policies}

We begin by tuning the parameters of the $\epsilon$-greedy, Thompson sampling and $L-L_{m}$ round robin policies for each of the four scenarios to find the parameter values that yield the best results for our simulation setting.

1) Tuning $\epsilon$ : The values of $\epsilon$, denoted by $\epsilon_{\text {tuned }}$, over the interval $[0.001,0.5]^{14}$ that result in the smallest sum $P_{\text {out }}^{\Pi}$ over $K$ in our simulations are listed in Table III for the four scenarios. The performances for different $\epsilon$ can be seen in Figs. 4 and 5 .

\begin{tabular}{|l|l|l|l|l|}
\hline Beamforming/ Frequency & Analog/ $28 \mathrm{GHz}$ & Digital/ $28 \mathrm{GHz}$ & Analog/ $140 \mathrm{GHz}$ & Digital/ $140 \mathrm{GHz}$ \\
\hline$\epsilon_{\text {tuned }}$ & 0.00125 & 0.01 & 0.00125 & 0.005 \\
\hline
\end{tabular}

TABLE III: $\epsilon_{\text {tuned }}$ for different frequencies and beamforming schemes

\footnotetext{
${ }^{14}$ Over the 3000 SSB burst periods in each simulated trajectory, the probability that the $\epsilon$-greedy policy explores at least is $1-(1-\epsilon)^{3000} \approx 0.95$ for $\epsilon=0.001$. Hence, $\epsilon \geq 0.001$ provides a high probability for the $\epsilon$-greedy policy to explore at least once after convergence in our simulations. We restrict the upper limit of $\epsilon$ to 0.5 , since we wish our policy to exploit what it has learnt at least half the time.
} 
2) Choice of $\zeta_{n}$ for Thompson sampling: As discussed in Section IV-C, the performance of Thompson sampling depends on the choice of $\zeta_{n}$ and the resulting $f\left(X_{n} \mid \mathcal{G}_{n}(t)\right)$ for the average link SNRs. We consider three distinct models from the exponential family for the likelihood function, $f\left(\mathcal{G}_{n}(t) \mid X_{n}\right)$, with each model corresponding to a conjugate prior choice for $\zeta_{n}$ :

(i) Gaussian $\zeta_{n}$, assuming Gaussian $f\left(\mathcal{G}_{n}(t) \mid X_{n}\right)$,

(ii) Gamma $\zeta_{n}$, assuming exponential $f\left(\mathcal{G}_{n}(t) \mid X_{n}\right)$, and

(iii) Beta $\zeta_{n}$ : Instead of the average SNR, the outage probability of the individual links can also be used to determine the tracking set. Let $\theta_{n} \in(0,1)$ denote the outage probability of the $n$-th link. Given $\mathcal{G}_{n}(t)$, let $\mathbf{g}_{n}(t)$ denote the binary vector (of size $\hat{N}_{n}(t)$ ) obtained by mapping the measured SNRs above and below $\gamma_{\text {tgt }}$ to 0 and 1 , respectively. Thus, $\mathbf{g}_{n}(t)$ captures the instances (up to the $t$-th SSB burst period) when the $n$-th link is measured to be in outage. Since $\mathbf{g}_{n}(t)$ is a sufficient statistic for estimating $\theta_{n}$, Algorithm 3 can be used to determine $\mathcal{A}(t)$ by replacing $X_{n}$ with $\theta_{n}$ and $\mathcal{G}_{n}(t)$ with $\mathbf{g}_{n}(t)$, respectively. As $\mathbf{g}_{n}(t)$ takes values in $\{0,1\}$, we assume Bernoulli $f\left(\mathbf{g}_{n}(t) \mid \theta_{n}(t)\right)$, leading to Beta $\zeta_{n}$.

Since the above conjugate priors are completely specified by two parameters, updating the posterior distribution $f\left(X_{n} \mid \mathcal{G}_{n}(t)\right)$ in Algorithm 3 reduces to a simple update rule for the parameters at each $t$, as given by Table IV [38].

We denote by $\zeta_{\text {tuned }}$ the prior yielding the smallest sum $P_{\text {out }}^{\Pi}$ over $K$ among the choices above

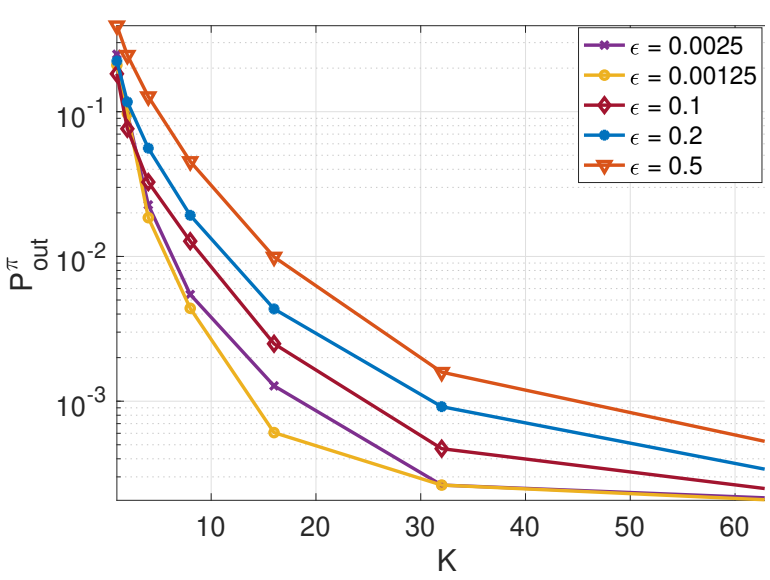

(a) Analog beamforming

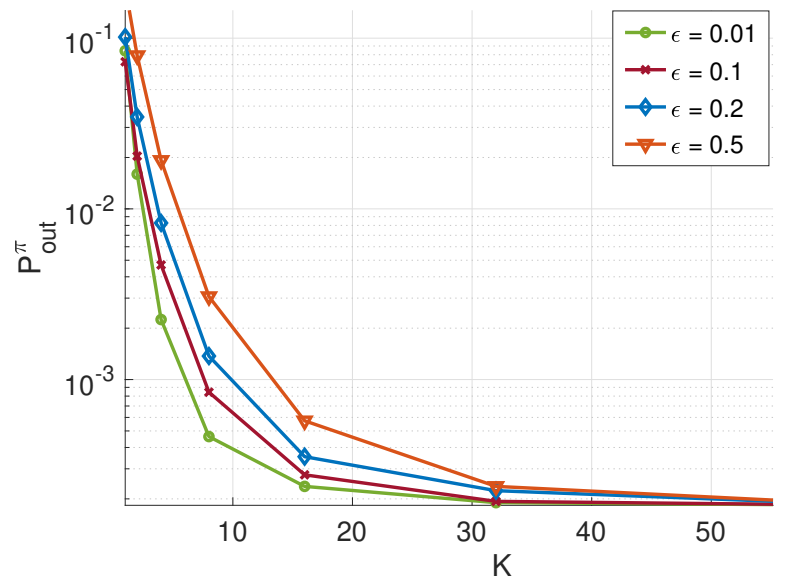

(b) Digital beamforming

Fig. 4: $P_{\mathrm{out}}^{\Pi}$ as a function of $K$ for the $\epsilon$-greedy link tracking policy for different values of $\epsilon$ at $28 \mathrm{GHz}$. 


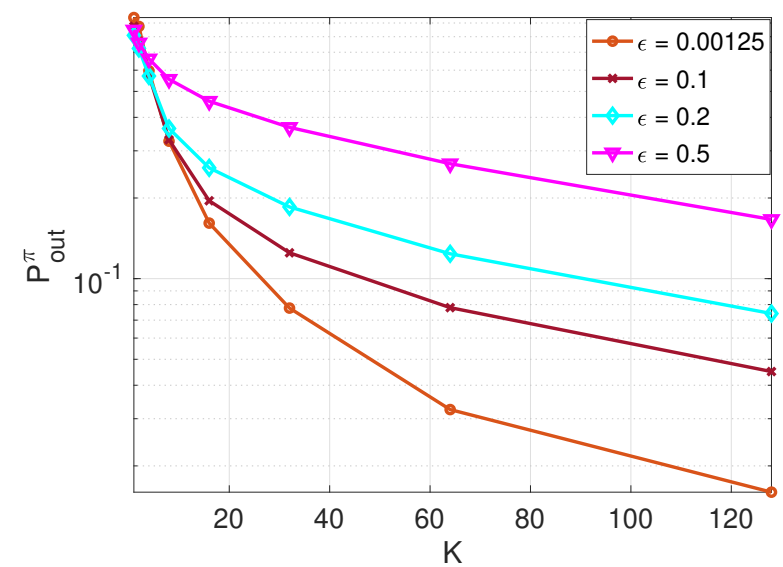

(a) Analog beamforming

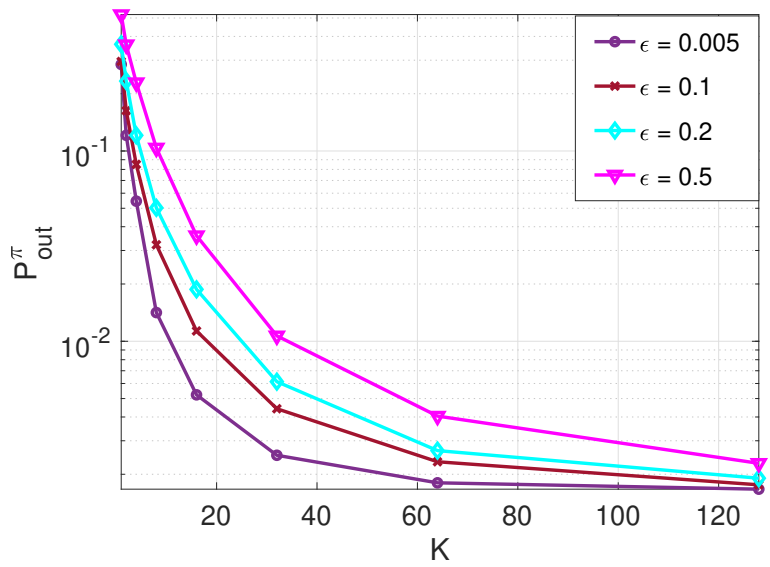

(b) Digital beamforming

Fig. 5: $P_{\text {out }}^{\Pi}$ as a function of $K$ for the $\epsilon$-greedy link tracking policy for different values of $\epsilon$ at $140 \mathrm{GHz}$.

\begin{tabular}{|c|c|c|}
\hline Prior & $\zeta_{n}$ & Posterior \\
\hline Gaussian & $\mathcal{N}(0,1)$ & $f\left(X_{n} \mid \mathcal{G}_{n}(t)\right) \sim \mathcal{N}\left(\bar{\gamma}_{n}(t), 1 / \hat{N}_{n}(t)\right)$ \\
\hline Gamma & $\operatorname{Gamma}(1,1)$ & $f\left(X_{n} \mid \mathcal{G}_{n}(t)\right) \sim \operatorname{Gamma}\left(1+\hat{N}_{n}(t), 1+\hat{N}_{n}(t) \bar{\gamma}_{n}(t)\right)$ \\
\hline Beta & $\operatorname{Beta}(1,1)$ & $\left.f\left(\theta_{n} \mid \mathbf{g}_{n}(t)\right) \sim \operatorname{Beta}\left(1+\mathbf{1}^{T} \mathbf{g}_{n}(t), 1+\hat{N}_{n}(t)-\mathbf{1}^{T} \mathbf{g}_{n}(t)\right)\right)$ \\
\hline
\end{tabular}

TABLE IV: Parameter updates for the posterior distribution of $X_{n}$ or $\theta_{n}$, corresponding to different conjugate prior choices for $\zeta_{n}$, for the Thompson Sampling link tracking policy in Algorithm 3.

in our simulations. $\zeta_{\text {tuned }}$ for each case is listed in Table V. Performances for different $\zeta$ can be observed in Fig. 6.

\begin{tabular}{|l|l|l|l|l|}
\hline Beamforming/ Frequency & Analog/ $28 \mathrm{GHz}$ & Digital/ $28 \mathrm{GHz}$ & Analog/ $140 \mathrm{GHz}$ & Digital/ $140 \mathrm{GHz}$ \\
\hline$\zeta_{\text {tuned }}$ & Gaussian Prior & Beta Prior & Beta Prior & Beta Prior \\
\hline
\end{tabular}

TABLE V: $\zeta_{\text {tuned }}$ for different frequencies and beamforming schemes

3) Tuning $L_{m}$ : Over $1 \leq L_{m}<L$, we observed that increasing $L_{m}$ leads to lower $P_{\text {out }}^{\Pi}$ for a given $K$, but we did not observe any significant improvement for $L_{m}>\frac{3 L}{4}$. Hence, we consider $L_{m}=3 L / 4$ for each case from here on. The performance of the policy with different values of $L_{m}$ can be seen in Fig. 7 and 8.

\section{B. Outage Performance of Policies}




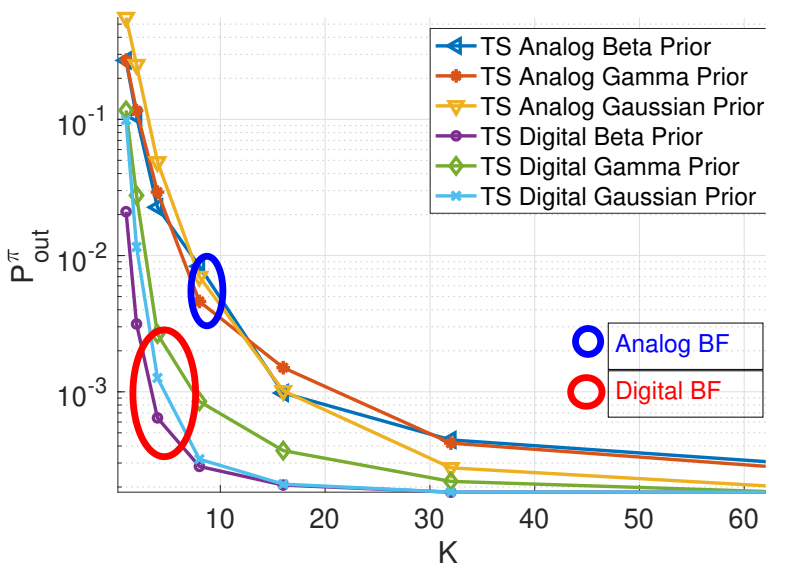

(a) $28 \mathrm{GHz}$

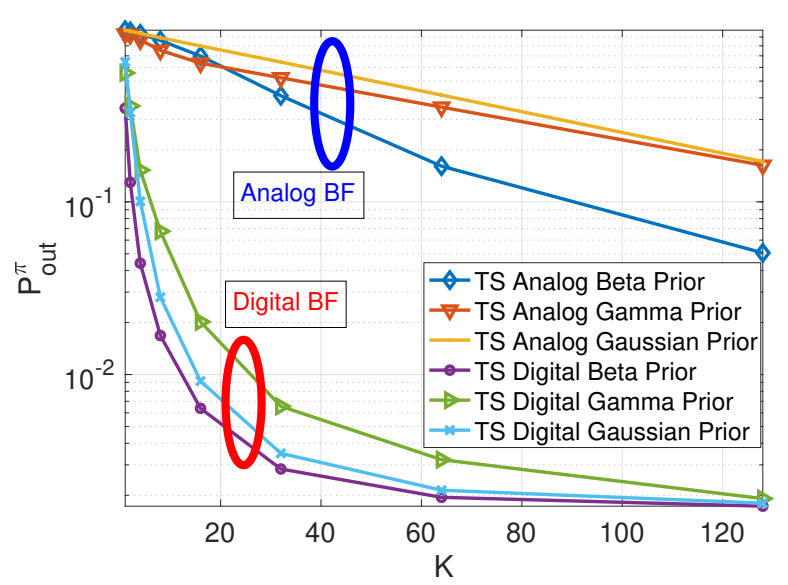

(b) $140 \mathrm{GHz}$

Fig. 6: $P_{\text {out }}^{\Pi}$ as a function of $K$ for the Thompson sampling (TS) link tracking policy for different prior distributions $\zeta$ at 28 and $140 \mathrm{GHz}$ with analog and digital beamforming.

We proceed to compare the outage performance of the tuned policies in connected mode DRX against a "Genie" benchmark, which represents the ideal case where the UE knows (and thus, tracks) the link with the highest SNR at each SSB burst period. Let $\gamma_{\mathrm{g}}(t)$ denote the genie SNR during the $t$-th SSB burst, which is given by

$$
\gamma_{\mathrm{g}}(t):=\max _{i, l, k} \gamma_{i l k}(t)
$$

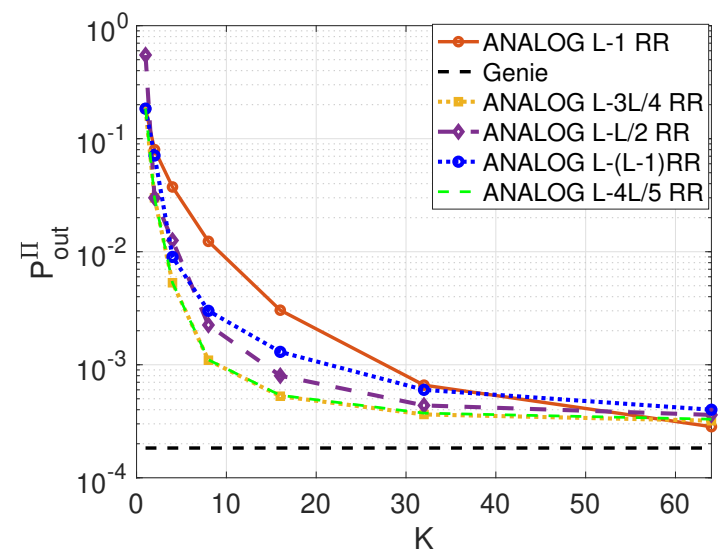

(a) Analog beamforming

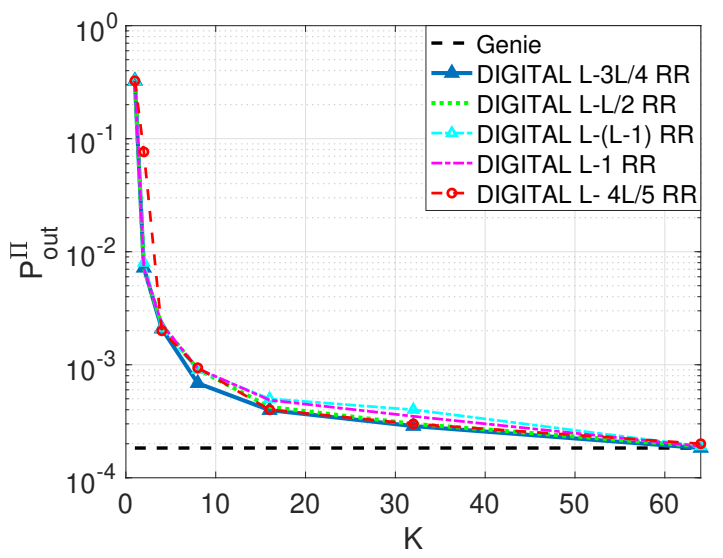

(b) Digital beamforming

Fig. 7: $P_{\text {out }}^{\Pi}$ as a function of $K$ for the $L-L_{m}$ RR link tracking policy for different values of $L_{m}$ at $28 \mathrm{GHz}$. 


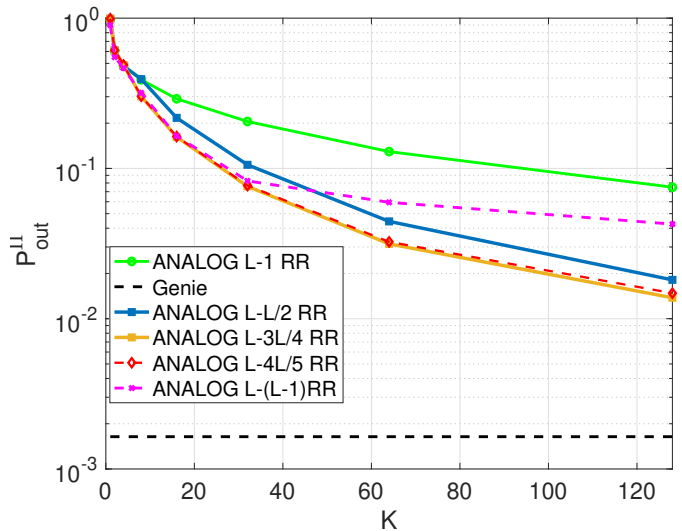

(a) Analog beamforming

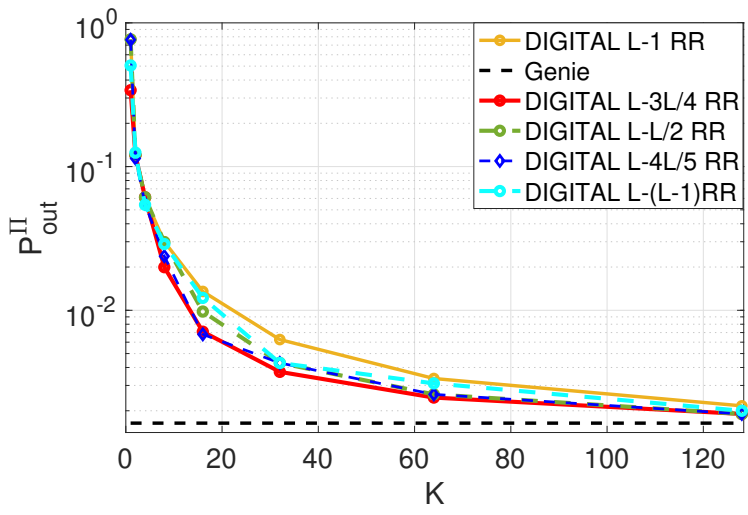

(b) Digital beamforming

Fig. 8: $P_{\mathrm{out}}^{\Pi}$ as a function of $K$ for the $L-L_{m}$ RR link tracking policy for different values of $L_{m}$ at $140 \mathrm{GHz}$.

Similar to (22), the genie outage probability, denoted by $P_{\text {out }}^{\mathrm{g}}$, has the following expression:

$$
P_{\mathrm{out}}^{\mathrm{g}}:=\mathbb{P}\left(\gamma_{\mathrm{g}}(t)<\gamma_{\text {tgt }}\right)
$$

At $28 \mathrm{GHz}$ with analog beamforming, we see from Fig. 9a that $K=8$, which translates to the UE being awake for only $12.5 \%$ of the time during an SSB burst period, results in an outage probability of less than $1 \%$ for all policies. For digital beamforming, the power savings are

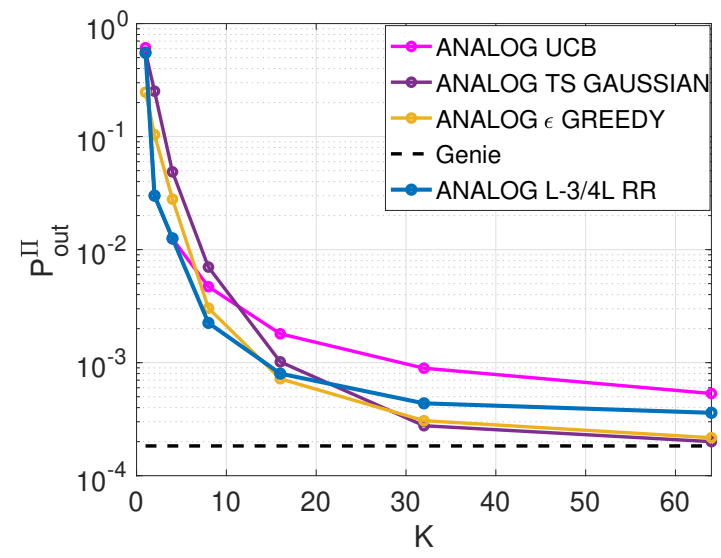

(a) Analog beamforming

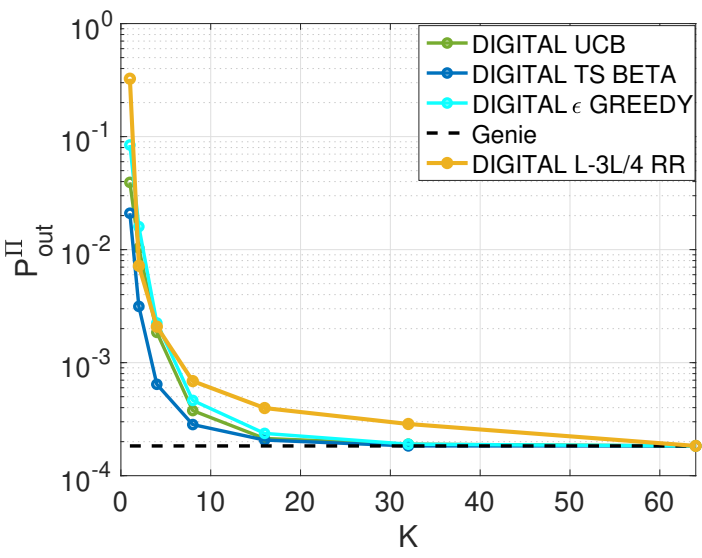

(b) Digital beamforming

Fig. 9: $P_{\text {out }}^{\Pi}$ as a function of $K$ for different policies at $28 \mathrm{GHz}$. 


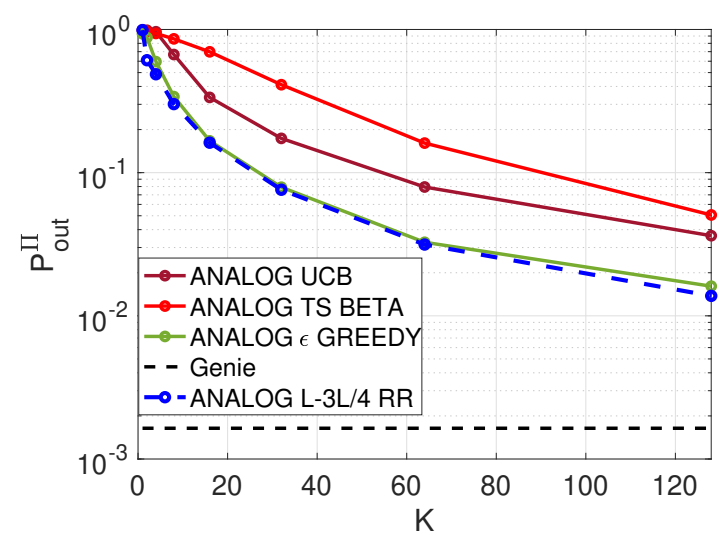

(a) Analog beamforming

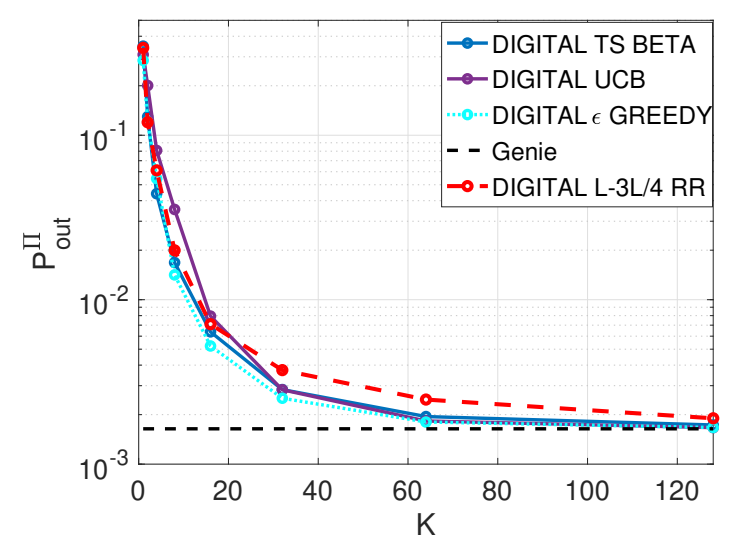

(b) Digital beamforming

Fig. 10: $P_{\text {out }}^{\Pi}$ as a function of $K$ for different policies at $140 \mathrm{GHz}$.

greater as $K=4$ (i.e., $6.25 \%$ awake time) is sufficient to achieve the same outage performance for all the policies (see Fig. 9b).

At $140 \mathrm{GHz}$, we see from Fig. 10 that the performance gap between analog and digital beamforming is very large, compared to $28 \mathrm{GHz}$. In particular, for analog beamforming with $K=N_{\mathrm{SSB}}=128$ (i.e., the UE is awake for the whole SSB burst period), the outage probability is still more than $1 \%$ for all the link tracking policies (Fig. 10a). On the other hand, for digital beamforming, $K=16$ (i.e., the UE can sleep for $87.5 \%$ of the time during an SSB burst period) results in an outage probability of less than $1 \%$ for all policies (see Fig. 10b). We make the following remarks on the poor performance of the policies for the analog beamforming case at $140 \mathrm{GHz}$.

Remark 6 (Poor outage performance of policies for analog beamforming at $140 \mathrm{GHz}$ ). Let $\kappa$ denote the fraction of links the UE tracks when $K=N_{\mathrm{SSB}}$ (i.e., $L=L_{\max }$ from Remark 4), which is given by:

$$
\kappa=\frac{L_{\mathrm{max}}}{N_{\mathrm{cell}} N_{\mathrm{TX}} N_{\mathrm{RX}}}= \begin{cases}\frac{N_{\mathrm{SSB}}}{N_{\mathrm{TX}} N_{\mathrm{RX}}}, & \text { for Analog beamforming, } \\ \frac{N_{\mathrm{SSB}}}{N_{\mathrm{TX}}}, & \text { for Digital beamforming }\end{cases}
$$

The value of $\kappa$ for the four cases is listed in Table VI, where we observe that for analog beamforming at $140 \mathrm{GHz}$, the UE tracks a significantly smaller fraction of the total number of links compared to the other cases. Hence, even for $K=N_{\mathrm{SSB}}$, the probability of tracking the 
link with the highest SNR (or even a good link, for that matter) is considerably smaller than the other cases, which explains the poor performance.

\begin{tabular}{|l|l|l|l|l|}
\hline Beamforming/ Freq. & Analog/ $28 \mathrm{GHz}$ & Digital/ $28 \mathrm{GHz}$ & Analog/ $140 \mathrm{GHz}$ & Digital/ $140 \mathrm{GHz}$ \\
\hline$\kappa$ & $1 / 8$ & 1 & $1 / 132$ & $1 / 2$ \\
\hline
\end{tabular}

TABLE VI: $\kappa$ for different frequencies and beamforming schemes

Remark 7 (Implication for standardization efforts at $140 \mathrm{GHz}$ ). From (25) and Tables VI and $I X$, it is easy to see that the small value of $\kappa$ for analog beamforming at $140 \mathrm{GHz}$ is due to $N_{\mathrm{SSB}}$ (which is fixed by the $3 G P P N R$ standards at $28 \mathrm{GHz}$ ) not scaling at the same rate as $N_{\mathrm{TX}} N_{\mathrm{RX}}$ from 28 to $140 \mathrm{GHz}$. A larger value of $N_{\mathrm{SSB}}$, that is commensurate with the array gain $\left(N_{\mathrm{TX}} N_{\mathrm{RX}}\right)$ scaling needed to overcome pathloss, would improve the policies' performance in Fig. 10a, but at the cost of increased power consumption. Significant standardization efforts, involving parameters like the sub-carrier spacing, bandwidth etc. (see the Remarks column of Table IX for more details) are needed, to identify the right value(s) of $N_{\mathrm{SSB}}$ at $140 \mathrm{GHz}$, in order to ensure the viability of analog beamforming (in terms of both power-efficiency and performance) at the UE.

The winning policy in our simulations for each case, defined as the one with smallest sum $P_{\text {out }}^{\Pi}$ across $K$, is reported in Table VII, and is used to analyze the power versus throughput trade-off in the next subsection.

\begin{tabular}{|l|l|l|l|l|}
\hline Beamforming/ Frequency & Analog/ $28 \mathrm{GHz}$ & Digital/ $28 \mathrm{GHz}$ & Analog/ $140 \mathrm{GHz}$ & Digital/ $140 \mathrm{GHz}$ \\
\hline Winning Policy & $\epsilon$-greedy & Thompson sampling & $L-3 / 4 L$ Round Robin & $\epsilon$-greedy \\
\hline
\end{tabular}

TABLE VII: Winning policies in our simulations.

\section{Power vs. Throughput Trade-off}

In this section, we formulate an optimization problem that captures the trade-off between the UE power consumption and throughput. We use the spectral efficiency $\eta$, of the MCS levels 
to map $\gamma_{\max }^{\Pi}$ to throughput ${ }^{15}$ from [46, Table 1]. We consider MCS 0-28 $(u=0 \ldots 28)$ for downlink communications from 3GPP standard. Let $\eta_{u}$ be the spectral efficiency of $u$-th MCS. Let $\hat{\gamma}_{u}$ denote the minimum SNR need to decode MCS $u$, given by:

$$
\widehat{\gamma}_{u}:=\Delta\left(2^{\eta_{u}}-1\right)
$$

The loss factor $\Delta$, is a measure of how far the system is operating from Shannon capacity. The value of $\Delta=2(3 \mathrm{~dB})$ is in accordance with [5], [48], [56]. We define $p_{u K}$ as the probability that MCS $u$ is supported when the UE is awake for $K$ SSBs i.e.,

$$
p_{u K}:=\mathbb{P}\left(\gamma_{\max }^{\Pi} \geq \widehat{\gamma}_{u}\right)
$$

The heat maps in Figs. 11 and 12 plot $p_{u K}$ for the winning policies in Table VII, with $K \in$ $\{1,2,4,8,16,32,64\}$ for $28 \mathrm{GHz}$ and $K \in\{1,2,4,8,16,32,64,128\}$ for $140 \mathrm{GHz}$. For a fixed $u$ (i.e., fixed MCS/throughput), $p_{u K}$ increases with increasing $K$, as there is a greater chance that the UE can discover a better link if it awake for longer. This is seen in Figs. 11 and 12, where for a given row (e.g., $u=15$ ), as the value of $K$ increases, the color transitions from cold to hot, signaling an increase in $p_{u K}$. On the other hand, for a fixed $K$, as $u$ increases, $p_{u K}$ decreases because the minimum SNR required to decode a higher MCS [i.e., the threshold $\hat{\gamma}_{u}$ in (27)] is greater; for instance, in Figs. 11 and 12, for a given column ( $K=8$, say), as $u$ increases, the color transitions from hot to cold, capturing a decrease in $p_{u K}$.

From $p_{u K}$, the expected spectral efficiency $\mathbb{E}\left[\eta_{u K}\right]$ for MCS $u$ and awake time $K$ can be expressed as:

$$
\mathbb{E}\left[\eta_{u K}\right]=p_{u K} \eta_{u}
$$

We can now capture the power-throughput trade-off using the following optimization problem:

$$
\begin{gathered}
\underset{u, K}{\arg \max } \mathbb{E}\left[\eta_{u K}\right]-\delta K \\
\text { s.t. } p_{u K} \geq P_{o} .
\end{gathered}
$$

In (29), $\delta>0$ is a tunable parameter that can be used to penalize power consumption, e.g., for a UE operating in low-power mode, a large $\delta$ is appropriate, whereas for a fully charged device anticipating high throughput traffic, a low $\delta$ may be suitable. The constraint (30) ensures that the optimal solution supports the chosen MCS with a minimum probability of $P_{o}$.

\footnotetext{
${ }^{15}$ Since the UE is in the pre-data arrival phase, this is, strictly speaking, the anticipated throughput based on $\gamma_{\max }^{\Pi}$, in case there is data arrival. For convenience, we continue to refer to it as throughput in the paper.
} 


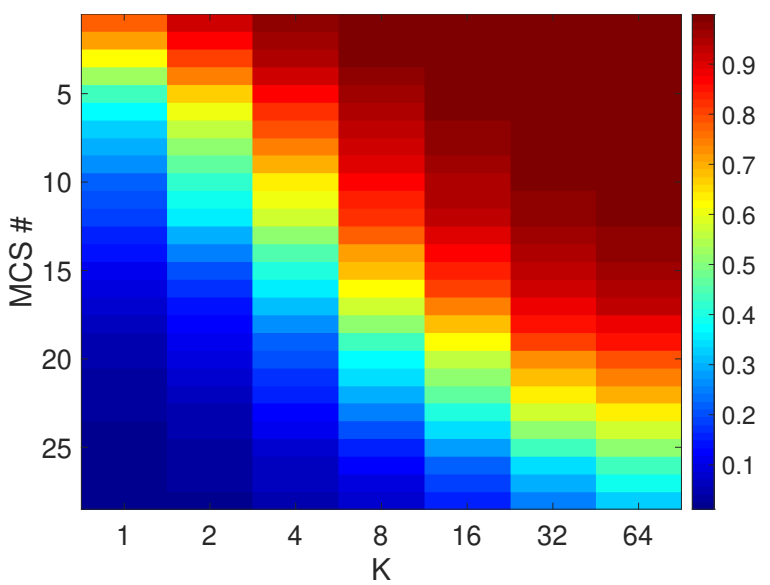

(a) Analog beamforming

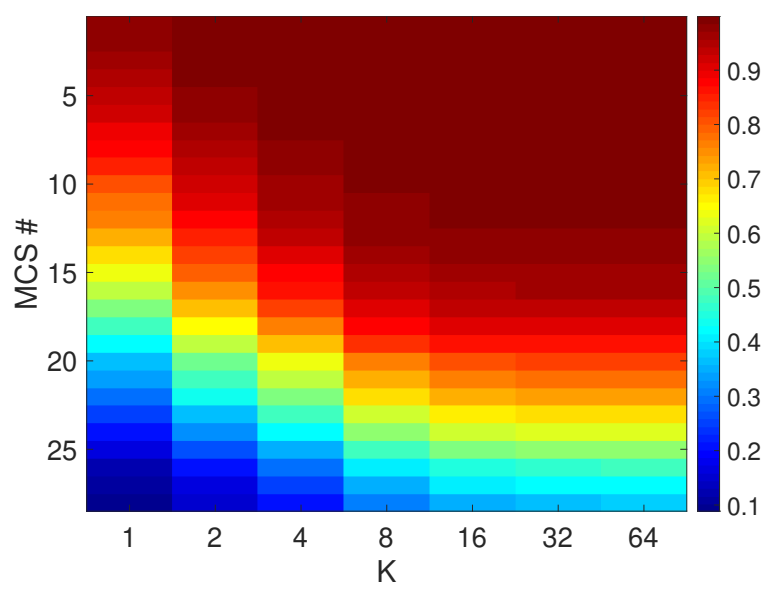

(b) Digital beamforming

Fig. 11: Heat map of $p_{u K}$ for analog and digital beamforming at $28 \mathrm{GHz}$

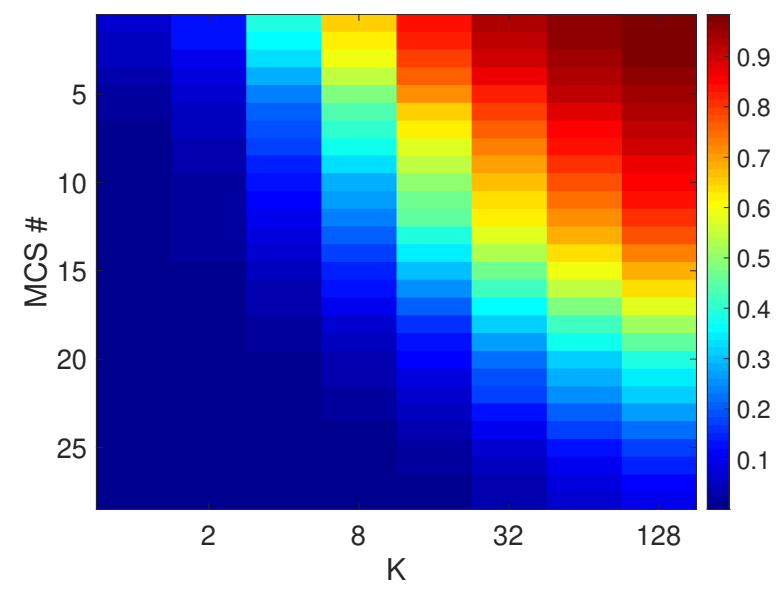

(a) Analog beamforming

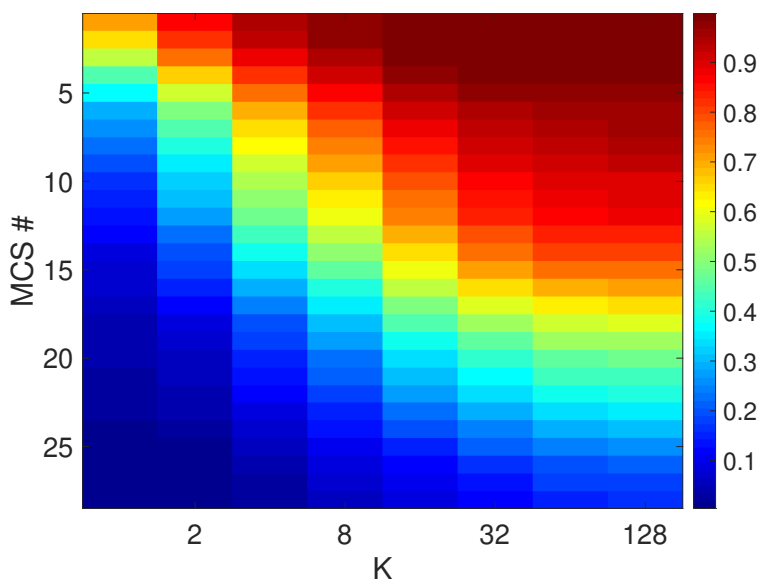

(b) Digital beamforming

Fig. 12: Heat map of $p_{u K}$ for analog and digital $\mathrm{BF}$ at $140 \mathrm{GHz}$

The choice of $\delta$ determines the operating point on the Pareto boundary of the power-throughput trade-off curve, which is shown for analog and digital beamforming at 28 and $140 \mathrm{GHz}$ in Fig. 13 for $P_{o}=99 \%$. The curve corresponding to analog beamforming at $140 \mathrm{GHz}$ is missing in Fig. 13b, since the optimization problem in (29)-(30) is infeasible for this case for $P_{o}=99 \%$, as seen in Fig. 10. The trade-off at the feasible 'knee-points' are presented in Table VIII.

Remark 8 (Implications of Table VIII). From an engineering perspective, Table VIII enables us to draw mild, but useful, achievability conclusions like "a well-designed link tracking policy 


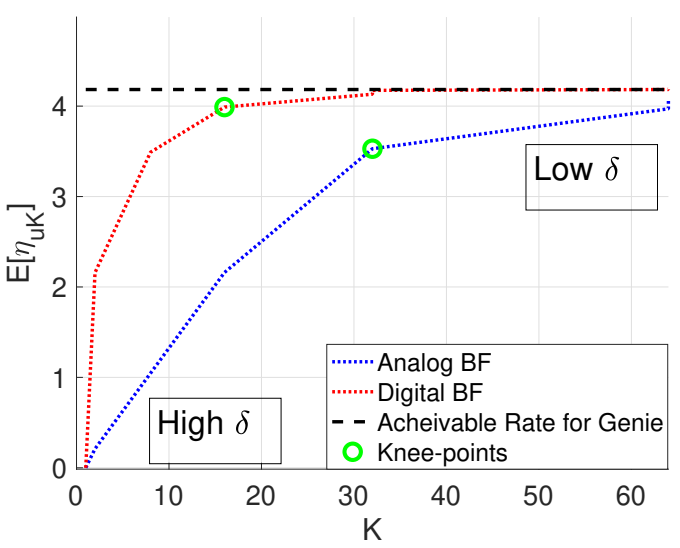

(a) $28 \mathrm{GHz}$

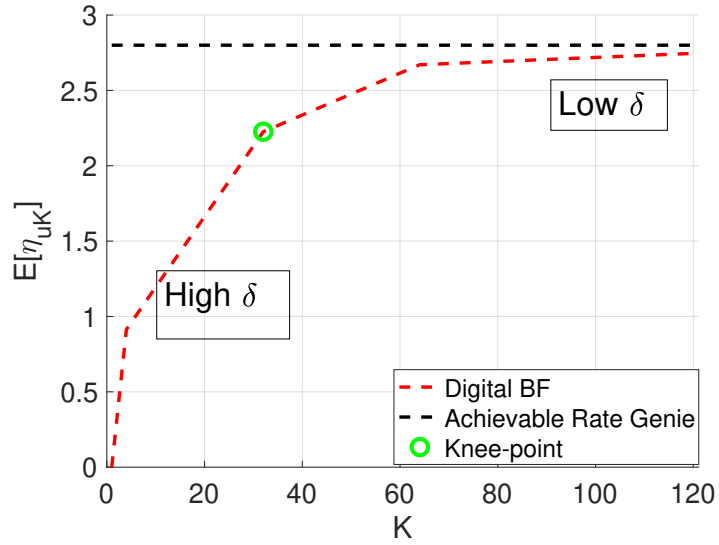

(b) $140 \mathrm{GHz}$

Fig. 13: Pareto boundaries for analog and digital beamforming at 28 and $140 \mathrm{GHz}$. We see that the $28 \mathrm{GHz}$ system achieves a peak spectral efficiency of $4.2 \mathrm{bps} / \mathrm{Hz}$, which reduces to $2.8 \mathrm{bps} / \mathrm{Hz}$ for $140 \mathrm{GHz}$. This is due to the higher blockage and reflection loss at sub-THz frequencies.

\begin{tabular}{|c|c|c|c|c|c|}
\hline $\begin{array}{c}\text { Beamforming/ } \\
\text { Frequency }\end{array}$ & $\begin{array}{c}\text { Knee Point } \\
\text { Power Saved } \\
\left.\text { (relative to } K=N_{\mathrm{SSB}}\right)\end{array}$ & $\begin{array}{l}\text { Achieved } \\
\text { Throughput } \\
\text { (relative to Genie) }\end{array}$ & $\begin{array}{l}\text { Power } \\
\text { Consumed } \\
(\mathbf{m W})\end{array}$ & $\begin{array}{l}\text { Empirical } \\
\text { Convergence } \\
\text { time } \\
\text { (SSB bursts) }\end{array}$ \\
\hline Analog/ $28 \mathrm{GHz}$ & 32 & $50 \%$ & $85 \%$ & 12.48 & 15 \\
\hline Digital/ $28 \mathrm{GHz}$ & 16 & $75 \%$ & $95 \%$ & 2.72 & 4 \\
\hline Analog/ $140 \mathrm{GHz}$ & \multicolumn{2}{|c|}{ Optimization problem in (29)-(30) is infeasible for $P_{\mathrm{o}}=99 \%$ even for MCS 0. This is } \\
equivalent to an outage probability greater than $1 \%$, which is consistent with Fig. 10a.
\end{tabular}

TABLE VIII: Power-throughput trade-off at the knee points in Fig. 13.

should simultaneously realize $50 \%$ power savings, $85 \%$ of the maximum throughput, and $<1 \%$ outage probability in a $5 \mathrm{G} \mathrm{mmWave} \mathrm{environment} \mathrm{at} 28 \mathrm{GHz}$ with analog beamforming at the UE" (and so on, for the other cases), since these benchmarks are achieved by the winning policy among a small collection of sub-optimal policies. In our opinion, conclusions like these are the key contributions of this paper. 


\section{Closeness to Optimality and Convergence Time}

While Fig. 13 provides an insight into the average throughput that can be achieved by the policies in Table VII, it does not indicate how closely and quickly their tracking performance approaches that of Genie.

In Fig. 14, we plot the CDFs of $\gamma_{\max }^{\Pi}$ corresponding to the knee points labelled in Fig. 13, along with the Genie CDF. At $28 \mathrm{GHz}$, digital beamforming with $K=16$ differs from Genie by only about $0.1 \mathrm{~dB}$ at 50 -th percentile, while for analog beamforming with $K=32$, the difference goes up to $2 \mathrm{~dB}$. However, the power consumed by analog beamforming in this case exceeds that of digital beamforming by a factor of 4.5, as shown in Table VIII. At $140 \mathrm{GHz}$, the difference at the 50 -th percentile is around $2 \mathrm{~dB}$ for digital beamforming $(K=32)$.

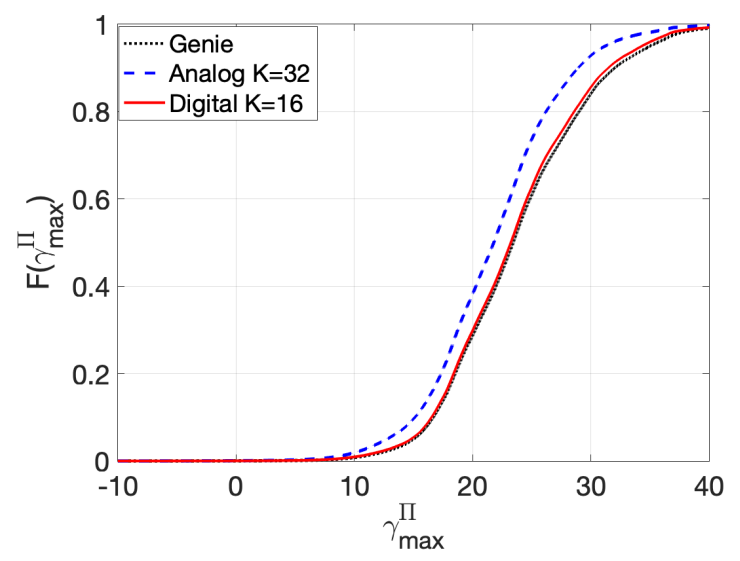

(a) $28 \mathrm{GHz}$

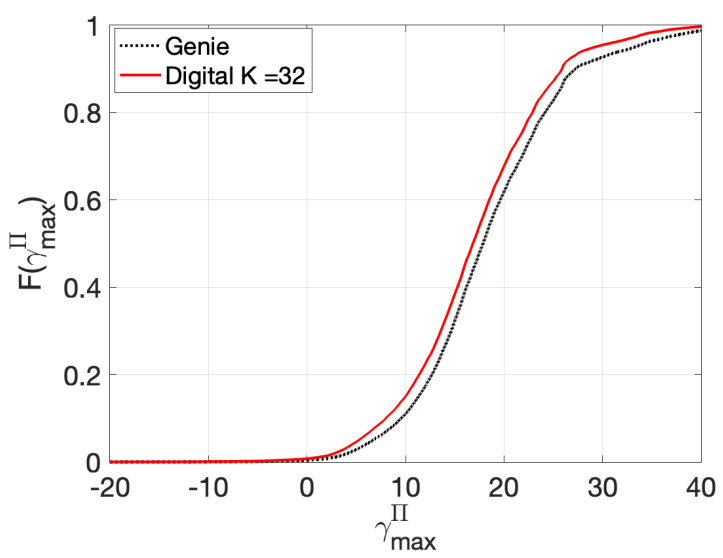

(b) $140 \mathrm{GHz}$

Fig. 14: CDFs of $\gamma_{\max }^{\Pi}$ corresponding to the knee points in Table VIII.

We define the convergence time of a policy $\Pi$ as the number of SSB bursts required for the ratio $\mathbb{E}\left[\gamma_{\mathrm{g}}^{\Pi}(t) / \gamma_{\max }^{\Pi}(t)\right]$ to be less than $3 \mathrm{~dB}$, where $\gamma_{\mathrm{g}}(t)$ and $\gamma_{\max }^{\Pi}(t)$ are given by (23) and (4), respectively, and the averaging is across simulation trajectories. The convergence behavior of the winning policies in Table VII is shown in Fig. 15, where we see that convergence is faster with digital beamforming due to the larger fraction of links that it permits the UE to track for a given $K$ (Remark 6). The convergence times from Fig. 15, in terms of the number of SSB burst periods, are listed in Table VIII. 


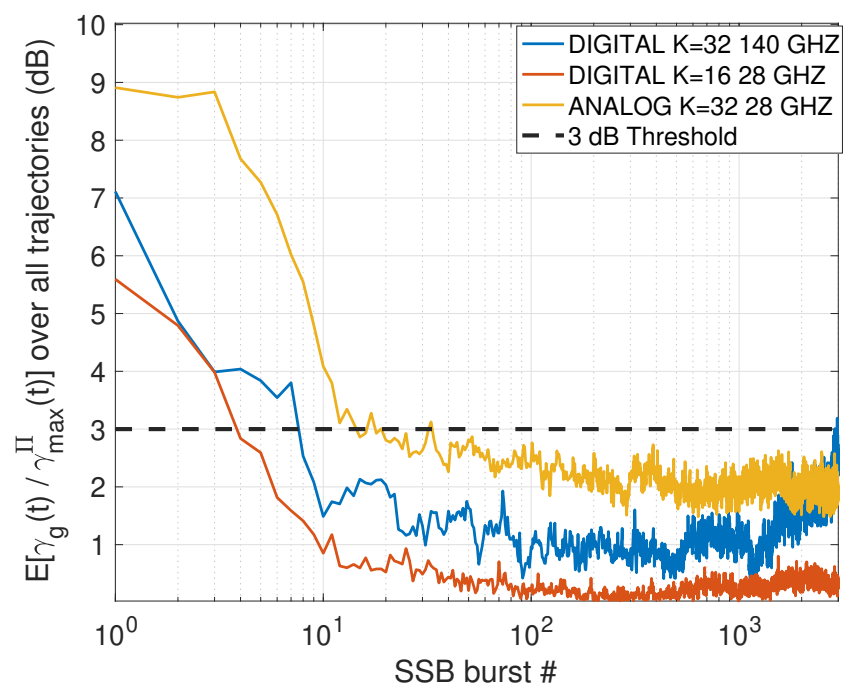

Fig. 15: Empirical convergence time of the winning policies from Table VII.

\section{E. Significance of Simulation Results}

- In Section II, we demonstrated the power intensive nature of beam tracking within connected mode DRX - a mechanism intended to reduce UE power consumption - for mmWave and sub-THz systems at $28 \mathrm{GHz}$ and $140 \mathrm{GHz}$, respectively (Table I and Remark 2). This is a critical issue from an energy efficiency perspective, which is a key performance metric for $5 \mathrm{G}$ and $6 \mathrm{G}$ systems [57], [58]. In this context, our results on the magnitude of the UE power savings that can be achieved $(50 \%-75 \%)$ without a large degradation in throughput (based on Table VIII and Remark 8) is significant, considering the scale of UEs (billions of devices) expected to be supported by a $5 \mathrm{G} / 6 \mathrm{G}$ network.

- Implications for Beamforming Architecture: From Table VIII, we observe that a digital beamforming architecture permits more power-efficient link tracking with lesser throughput degradation than analog beamforming, which is due to the former enabling the UE to track a larger fraction of the available links for any $K$, according to (3). This adds to the compelling case for digital beamforming at $28 \mathrm{GHz}$ made in [5], and extends it to $140 \mathrm{GHz}$, provided the advantages of digital beamforming with low-resolution ADCs extend to $140 \mathrm{GHz}$ as well (Remark 1).

With respect to analog beamforming, the especially poor performance of the policies at $140 \mathrm{GHz}$ is due to the extremely small fraction of the available links that the UE is permitted 
to track at each SSB burst period (Remark 6); hence, it is unlikely that a superior policy would perform any better. From Remark 7 , we see that this is due to $N_{\mathrm{SSB}}$ (the number of links that the UE is permitted to track) not increasing to an extent commensurate to the pathloss-driven scaling of $N_{\mathrm{TX}} N_{\mathrm{RX}}$ (the beamforming gain) from $28 \mathrm{GHz}$ to $140 \mathrm{GHz} . N_{\mathrm{SSB}}$ is determined by the 3GPP NR standards at $28 \mathrm{GHz}$, and we make reasonable assumptions for its value at $140 \mathrm{GHz}$ based on the standards (see the Remarks column corresponding to $N_{\mathrm{SSB}}$ in Table IX). Hence, for analog beamforming to be viable at $140 \mathrm{GHz}$ in terms of power-efficiency and outage/throughput performance, significant standardization efforts are needed to identify the appropriate value(s) of $N_{\mathrm{SSB}}$ for $140 \mathrm{GHz}$.

- Apart from the case of analog beamforming at $140 \mathrm{GHz}$, Table VIII suggests that the power versus performance trade-off can be favorably navigated using the sub-optimal policies considered in our paper. This is significant, since it suggests that considerable power savings can be realized even with unsophisticated policies. Thus, any of these policies could, in principle, be implemented for mmWave UEs with a short development cycle, leading to improved battery life on devices. However, a lot more work remains to be done to develop a policy that is well-suited for $5 \mathrm{G}$ mmWave and $6 \mathrm{G}$ THz systems with provable performance guarantees.

\section{SUMMARY}

DRX is likely to be aggressively used in mmWave and sub-THz wireless systems due to the high UE RFFE power consumption, which mainly stems from the need to track multiple links to ensure reliable multi-connectivity in the presence of frequent and severe link blockages. In this paper, we focused on reducing the UE power consumption during connected mode DRX by tracking only a subset of the available links, but without adversely affecting the outage/throughput performance. To achieve this objective, we formulated the choice of links to track over time as the outcome of a feasible policy for a MP-MAB problem. Through detailed system level simulations at 28 and $140 \mathrm{GHz}$, modeling a 5G mmWave and a hypothetical 6G sub-THz system, respectively, we observed that even sub-optimal link tracking policies could achieve considerable power savings with relatively little degradation in outage and throughput performance, especially with digital beamforming at the UE. 


\section{APPENDIX}

The UE RFFE components that contribute to the power terms on the right hand side of (1) are: (i) the Analog to Digital Converter(s) (ADCs), (ii) the Low Noise Amplifier(s) (LNAs), and (iii) the Local Oscillator (LO). We ignore the power consumption due to baseband processing as the procedures in question are not processing intensive. Let $P_{\mathrm{ADC}}^{f_{c}, \text { Analog }}$ and $P_{\mathrm{ADC}}^{f_{c} \text {, Digital denote }}$ the power consumed by the ADC at carrier frequency $f_{c}$ for analog and digital beamforming, respectively. Similarly, let $P_{\mathrm{LO}}^{f_{c}}$ and $P_{\mathrm{LNA}}^{f_{c}}$ denote the power consumed by the LOs and the LNAs, respectively.

From [10], the power consumed during the beam measurement (link tracking) procedure, $P_{\mathrm{BM}}^{f_{c}, \mathrm{BF}}$, is given by:

$$
P_{\mathrm{BM}}^{f_{c}, \mathrm{BF}}= \begin{cases}\frac{K T_{\mathrm{SSB}}}{T_{\mathrm{SS}}}\left(P_{\mathrm{ADC}}^{f_{c}, \text { Analog }}+P_{\mathrm{LO}}^{f_{c}}+P_{\mathrm{LNA}}^{f_{c}}\right), & \text { if } \mathrm{BF}=\text { Analog } \\ N_{\mathrm{RX}} \frac{K T_{\mathrm{SSB}}}{T_{\mathrm{SS}}}\left(P_{\mathrm{ADC}}^{f_{c}, \text { Digital }}+P_{\mathrm{LO}}^{f_{c}}+P_{\mathrm{LNA}}^{f_{c}}\right), & \text { if } \mathrm{BF}=\text { Digital. }\end{cases}
$$

We observe from (31) that $P_{\mathrm{BM}}^{f_{c}, \mathrm{BF}}$ has a linear dependence on $K$, which is proportional to the number of links tracked (see (3)). The additional $N_{\mathrm{RX}}$ factor for the digital beamforming case is a consequence of having $N_{\mathrm{RX}} \mathrm{RF}$ chains. The power consumed during assignment listening, $P_{\mathrm{LS}}^{f_{c}, \mathrm{BF}}$, can be written as:

$$
P_{\mathrm{LS}}^{f_{c}, \mathrm{BF}}= \begin{cases}\frac{T_{\mathrm{DRX}, \mathrm{ON}}}{T_{\mathrm{DRX}, \mathrm{Cyc}}}\left(P_{\mathrm{ADC}}^{f_{c}, \mathrm{Analog}}+P_{\mathrm{LO}}^{f_{c}}+P_{\mathrm{LNA}}^{f_{c}}\right), & \text { if } \mathrm{BF}=\text { Analog } \\ \frac{T_{\mathrm{DRX}, \mathrm{ON}}}{T_{\mathrm{DRX}, \mathrm{Cyc}}}\left(P_{\mathrm{ADC}}^{f_{c}, \text { Digital }}+P_{\mathrm{LO}}^{f_{c}}+P_{\mathrm{LNA}}^{f_{c}}\right), & \text { if } \mathrm{BF}=\text { Digital },\end{cases}
$$

where $T_{\mathrm{DRX}, \mathrm{ON}}$ is the DRX on time and $T_{\mathrm{DRX}, \mathrm{Cyc}}$ is the DRX cycle time. Finally, the power consumed in beam reporting, $P_{\mathrm{BR}}^{f_{c}, \mathrm{BF}}$, is given by:

$$
P_{\mathrm{BR}}^{f_{c}, \mathrm{BF}}= \begin{cases}\frac{2 T_{\mathrm{sym}}}{T_{\mathrm{DRX}, \mathrm{Cyc}}}\left(P_{\mathrm{ADC}}^{f_{c}, \text { Analog }}+P_{\mathrm{LO}}^{f_{c}}+P_{\mathrm{LNA}}^{f_{c}}\right), & \text { if } \mathrm{BF}=\text { Analog } \\ \frac{2 T_{\mathrm{sym}}}{T_{\mathrm{DRX}, \mathrm{Cyc}}}\left(P_{\mathrm{ADC}}^{f_{c}, \text { Digital }}+P_{\mathrm{LO}}^{f_{c}}+P_{\mathrm{LNA}}^{f_{c}}\right), & \text { if } \mathrm{BF}=\text { Digital },\end{cases}
$$

where $T_{\text {sym }}$ is the OFDM symbol period.

The quantities in (31)-(33) depend on many system parameters that depend on $f_{c}$, which are chosen according to the 3GPP NR standard and listed in Table IX. For further details, we refer the reader to [10]. 


\begin{tabular}{|c|c|c|c|}
\hline Parameter & \multicolumn{2}{|c|}{ Value } & Remarks \\
\hline Carrier frequency, $f_{c}$ & $28 \mathrm{GHz}$ & $140 \mathrm{GHz}$ & $\begin{array}{l}\text { The } 140 \mathrm{GHz} \text { band is the most likely sub-THz spectrum for } \\
\text { future } 6 \mathrm{G} \text { systems [8]. }\end{array}$ \\
\hline $\begin{array}{l}\text { Occupied bandwidth, } \\
B(\mathrm{GHz})\end{array}$ & 0.400 & 1.6 & $\begin{array}{l}\text { We assume that } B \text { would scale roughly with } f_{c} \text {. Thus, relative } \\
\text { to } 28 \mathrm{GHz} \text {, we consider a } 4 \times \text { increase in } B \text { at } 140 \mathrm{GHz} \text {. }\end{array}$ \\
\hline Sample rate $(\mathrm{GHz})$ & 0.491 & 1.966 & $\begin{array}{l}\text { The sample rate, which influences the ADC/DAC power } \\
\text { consumption, depends on } B \text { and the FFT size; } 28 \mathrm{GHz} \text { uses } \\
4096 \text { point FFT, while } 140 \mathrm{GHz} \text { could use } 2 \times 4096 \text { point FFT }\end{array}$ \\
\hline $\begin{array}{l}\text { Subcarrier spacing (SCS), } \\
\mathrm{kHz}\end{array}$ & 120 & 240 & $\begin{array}{l}\mathrm{SCS}=120 \mathrm{kHz} \text { and } B=400 \mathrm{MHz} \text { is common for early } 5 \mathrm{G} \\
\text { deployments at } 28 \mathrm{GHz} \text { [23]. The SCS is doubled at } 140 \mathrm{GHz} \text {, } \\
\text { since the bandwidth scales by a factor of } 4 \text {, while the FFT size } \\
\text { is only doubled. }\end{array}$ \\
\hline $\begin{array}{l}\text { OFDM symbol duration, } \\
T_{\mathrm{sym}}(\mu \mathrm{s})\end{array}$ & 8.92 & 4.46 & Derived from SCS [32]. \\
\hline $\begin{array}{l}\text { No. of UE (gNB) antennas, } \\
N_{\mathrm{RX}}\left(N_{\mathrm{TX}}\right)\end{array}$ & $8(64)$ & $64(256)$ & $\begin{array}{l}\text { The arrays sizes at } 28 \mathrm{GHz} \text { are similar to previous capacity } \\
\text { analyses [48], [59]. Assuming free space pathloss, } \\
N_{\mathrm{TX}} N_{\mathrm{RX}} \propto f_{c}^{2} \text { ensures a constant downlink power spectral } \\
\text { density at both carrier frequencies, so that the data rate scales } \\
\text { linearly with } B \text {. We consider a slightly larger scaling factor for } \\
N_{\mathrm{TX}} N_{\mathrm{RX}} \text { at } 140 \mathrm{GHz} \text { - } 32 \text { instead of } 25 \text { - since (a) for a UPA } \\
\text { at the } \mathrm{UE} \text { with } \lambda / 2 \text { element spacing, an eight-fold increase in } \\
N_{\mathrm{RX}} \text { results in a lower occupied chip area, and (b) a four-fold } \\
\text { increase in } N_{\mathrm{TX}} \text { is reasonable at the gNB. }\end{array}$ \\
\hline $\begin{array}{l}\text { Duration of one SSB, } T_{\mathrm{SSB}} \\
(\mu \mathrm{s})\end{array}$ & 35.7 & 17.9 & Equal to 4 OFDM symbols. \\
\hline SSB burst period, $T_{\mathrm{SS}}(\mathrm{ms})$ & 20 & 20 & Default NR configuration [32]. \\
\hline $\begin{array}{l}\text { Maximum no. of SSBs } \\
\text { during an SSB burst period } \\
N_{\mathrm{SSB}}\end{array}$ & 64 & 128 & $\begin{array}{l}N_{\mathrm{SSB}} \text { has a maximum value of } 64 \text { for } 5 \mathrm{G} \text { NR systems for the } \\
\text { default } 120 \mathrm{KHz} \text { SCS [32]. The } 240 \mathrm{kHz} \text { SCS at } 140 \mathrm{GHz} \\
\text { leads to a doubling of } N_{\mathrm{SSB}}[55] .\end{array}$ \\
\hline $\begin{array}{l}\text { DRX cycle time }(\mathrm{ms}) \\
T_{\mathrm{DRX}, \mathrm{Cyc}}\end{array}$ & 5 & 5 & From [60]. \\
\hline $\begin{array}{l}\text { DRX on time }(\mathrm{ms}) \\
T_{\mathrm{DRX}, \mathrm{ON}}\end{array}$ & $1 / 32$ & $1 / 32$ & \\
\hline
\end{tabular}

TABLE IX: 3GPP NR based system parameters used to estimate the UE RFFE power consumption in connected mode DRX. Similar parameters are used in [10]. 


\section{REFERENCES}

[1] S. H. Ali Shah, S. Aditya, S. Dutta, C. Slezak, and S. Rangan, "Power Efficient Discontinuous Reception in THz and mmWave Wireless Systems," in Proc. IEEE Intl. Workshop on Process. Adv. in Wireless Commun. (SPAWC), Jul. 2019, pp. 1-5.

[2] T. S. Rappaport, R. W. Heath Jr., R. C. Daniels, and J. N. Murdock, Millimeter Wave Wireless Communications. Pearson Education, 2014.

[3] T. S. Rappaport et al., "Millimeter wave mobile communications for 5G cellular: It will work!” IEEE Access, vol. 1, pp. 335-349, May 2013.

[4] S. Rangan, T. S. Rappaport, and E. Erkip, “Millimeter-Wave Cellular Wireless Networks: Potentials and Challenges,” Proc. IEEE, vol. 102, no. 3, pp. 366-385, Mar. 2014.

[5] S. Dutta, C. Barati, A. Dhananjay, D. A. Ramirez, J. F. Buckwalter, and S. Rangan, "A Case for Digital Beamforming at mmWave," IEEE Trans. Wireless Commun., Oct. 2019.

[6] T. Kürner and S. Priebe, "Towards THz communications-status in research, standardization and regulation," J. Infrared, Millimeter, and Terahertz Waves, vol. 35, no. 1, pp. 53-62, Aug. 2014.

[7] I. F. Akyildiz, J. M. Jornet, and C. Han, "Terahertz band: Next frontier for wireless communications," Physical Commun., vol. 12, pp. 16-32, Sep. 2014.

[8] Y. Xing and T. S. Rappaport, "Propagation measurement system and approach at $140 \mathrm{GHz}$-moving to $6 \mathrm{G}$ and above 100 GHz," in Proc. IEEE GLOBECOM, Dec. 2018, pp. 1-6.

[9] M. Giordani, M. Polese, M. Mezzavilla, S. Rangan, and M. Zorzi, “Toward 6G networks: Use cases and technologies," IEEE Commun. Mag., vol. 58, no. 3, pp. 55-61, Nov. 2020.

[10] P. Skrimponis et al., "Power Consumption Analysis for Mobile mmwave and Sub-THz Receivers," in 2020 IEEE 6G Wireless Summit, Mar. 2020, pp. 1-5.

[11] J. Zhou, N. Nikaein, and T. Spyropoulos, "LTE/LTE-A discontinuous reception modeling for machine type communications," IEEE Wireless Commun. Lett., vol. 2, no. 1, pp. 102-105, Dec. 2013.

[12] H. Ramazanali, "Performance evaluation of LTE/LTE-a DRX: A Markovian approach," IEEE Internet Things J., vol. 3, no. 3, pp. 386-397, Oct. 2016.

[13] 3GPP, “TS 38.300, NR and NG-RAN Overall Description; Stage 2,” Apr. 2020.

[14] —_ “TS 36.331 E-UTRA - Radio Resource Control (RRC) protocol specification - Release 13,” Jan. 2016.

[15] D. Liu, C. Wang, and L. K. Rasmussen, "Discontinuous Reception for Multiple-Beam Communication,” IEEE Access, vol. 7, pp. 46931-46946, Apr. 2019.

[16] S. Kwon, J. Hwang, A. Agiwal, and H. Kang, "Performance analysis of DRX mechanism considering analogue beamforming in millimeter-wave mobile broadband system," in 2014 IEEE Globecom Workshops (GC Wkshps), Mar. 2014, pp. 802-807.

[17] M. K. Maheshwari, M. Agiwal, N. Saxena, and A. Roy, "Hybrid directional discontinuous reception (HD-DRX) for 5G communication,” IEEE Commun. Lett., vol. 21, no. 6, pp. 1421-1424, Mar. 2017.

[18] M. Agiwal, M. Maheshwari, N. Saxena, and A. Roy, "Directional-DRX for 5G wireless communications," Electron. Lett., vol. 52, no. 21, pp. 1816-1818, Oct. 2016.

[19] C. Slezak, V. Semkin, S. Andreev, Y. Koucheryavy, and S. Rangan, "Empirical Effects of Dynamic Human-Body Blockage in $60 \mathrm{GHz}$ Communications,” IEEE Commun. Mag., vol. 56, no. 12, pp. 60-66, Dec. 2018.

[20] V. Raghavan et al., "Spatio-Temporal Impact of Hand and Body Blockage for Millimeter-Wave User Equipment Design at 28 GHz," IEEE Commun. Mag., vol. 56, no. 12, pp. 46-52, Dec. 2018. 
[21] G. R. MacCartney, T. S. Rappaport, and S. Rangan, "Rapid fading due to human blockage in pedestrian crowds at 5G millimeter-wave frequencies," in Proc. IEEE GLOBECOM, Jan. 2017, pp. 1-7.

[22] J. Choi, "On the macro diversity with multiple BSs to mitigate blockage in millimeter-wave communications," IEEE Commun. Lett., vol. 18, no. 9, pp. 1653-1656, Jul. 2014.

[23] M. Shafi et al., "5G: A tutorial overview of standards, trials, challenges, deployment, and practice," IEEE J. Sel. Areas Commun., vol. 35, no. 6, pp. 1201-1221, Apr. 2017.

[24] D. Corcoran, L. Andimeh, A. Ermedahl, P. Kreuger, and C. Schulte, "Data driven selection of DRX for energy efficient 5G RAN,” in 2017 13th Intl. Conf. on Netw. and Service Manag. (CNSM), Jan. 2017, pp. 1-9.

[25] L. Sharma, B. B. Kumar, and S. Wu, "Performance Analysis and Adaptive DRX Scheme for Dual Connectivity," IEEE Internet Things J., vol. 6, no. 6, pp. 10289-10304, Aug. 2019.

[26] M. Giordani and M. Zorzi, "Improved user tracking in $5 \mathrm{~g}$ millimeter wave mobile networks via refinement operations," in 2017 16th Annual Mediterranean Ad Hoc Networking Workshop (Med-Hoc-Net), Aug. 2017, pp. 1-8.

[27] M. Polese, M. Giordani, M. Mezzavilla, S. Rangan, and M. Zorzi, "Improved handover through dual connectivity in 5G mmwave mobile networks," IEEE J. Sel. Areas Commun., vol. 35, no. 9, pp. 2069-2084, Sep. 2017.

[28] J. Palacios, D. De Donno, and J. Widmer, "Tracking mm-wave channel dynamics: Fast beam training strategies under mobility," in IEEE INFOCOM 2017 - Proc. IEEE Comp. Commun., Oct. 2017, pp. 1-9.

[29] S. Jayaprakasam, X. Ma, J. W. Choi, and S. Kim, "Robust beam-tracking for mmwave mobile communications," IEEE Commun. Lett., vol. 21, no. 12, pp. 2654-2657, Sep. 2017.

[30] A. Alkhateeb, G. Leus, and R. W. Heath, "Compressed sensing based multi-user millimeter wave systems: How many measurements are needed?" in Proc. IEEE Intl. Conf. on Acoust., Speech, Signal Process. (ICASSP), Aug. 2015, pp. 2909-2913.

[31] Z. Xiao, T. He, P. Xia, and X.-G. Xia, "Hierarchical codebook design for beamforming training in millimeter-wave communication," IEEE Trans. Wireless Commun., vol. 15, no. 5, pp. 3380-3392, Jan. 2016.

[32] M. Giordani, M. Polese, A. Roy, D. Castor, and M. Zorzi, "A Tutorial on Beam Management for 3GPP NR at mmWave Frequencies," IEEE Commun. Surveys Tuts., Sep. 2018.

[33] G. Yuan, X. Zhang, W. Wang, and Y. Yang, "Carrier aggregation for LTE-advanced mobile communication systems," IEEE Commun. Mag., vol. 48, no. 2, pp. 88-93, Jan. 2010.

[34] Z. Shen, A. Papasakellariou, J. Montojo, D. Gerstenberger, and F. Xu, "Overview of 3GPP LTE-advanced carrier aggregation for 4G wireless communications," IEEE Commun. Mag., vol. 50, no. 2, pp. 122-130, Feb. 2012.

[35] 3GPP, "Requirements for support of radio resource management ," 3GPP TS 38.133 Release 15), Jul. 2019.

[36] J. Singh, O. Dabeer, and U. Madhow, "On the limits of communication with low-precision analog-to-digital conversion at the receiver," IEEE Trans. Commun., vol. 57, no. 12, pp. 3629-3639, Dec. 2009.

[37] V. Kuleshov and D. Precup, “Algorithms for multi-armed bandit problems,” J. Mach. Learn. Research, vol. 1, Feb. 2014.

[38] Y. Zhou, J. Zhu, and J. Zhuo, "Racing Thompson: an Efficient Algorithm for Thompson Sampling with Non-conjugate Priors," in Proc. Intl. Conf. on Mach. Learn., Jul. 2018, pp. 6000-6008. [Online]. Available: http://proceedings.mlr.press/v80/zhou18e.html

[39] Y. Gai, B. Krishnamachari, and R. Jain, "Combinatorial Network Optimization With Unknown Variables: Multi-Armed Bandits With Linear Rewards and Individual Observations," IEEE/ACM Trans. Netw., vol. 20, no. 5, pp. 1466-1478, Mar. 2012.

[40] V. Va and R. W. Heath, "Basic Relationship between Channel Coherence Time and Beamwidth in Vehicular Channels," in Proc. IEEE Veh. Technol. Conf. (VTC-Fall), Jan. 2015, pp. 1-5.

[41] 3GPP, “TR 38.901, Study on Channel Model for Frequencies From 0.5 to $100 \mathrm{GHz}$ (Release 15) document,” Jun. 2018. 
[42] V. Raghavan et al., "Millimeter-wave MIMO prototype: Measurements and experimental results," IEEE Commun. Mag., vol. 56, no. 1, pp. 202-209, Jan. 2018.

[43] T. Bai, R. Vaze, and R. W. Heath, "Analysis of Blockage Effects on Urban Cellular Networks," IEEE Trans. Wireless Commun., vol. 13, no. 9, pp. 5070-5083, Sep. 2014.

[44] METIS, “METIS Channel Model, Tech. Rep. METIS2020, Deliverable D1.4 v3.” Feb. 2015. [Online]. Available: https://www.metis2020.com/wp-content/uploads/deliverables/METIS D1.4 v1.0.pdf

[45] G. Maccartney, S. Deng, S. Sun, and T. Rappaport, "Millimeter-Wave Human Blockage at 73 GHz with a Simple Double Knife-Edge Diffraction Model and Extension for Directional Antennas," in Proc. IEEE Veh. Technol. Conf. (VTC-Fall), Mar. 2016.

[46] 3GPP, “TS 38.214 E-UTRA - 5G NR Physical layer procedures for data - Release 15,” Jan. 2020.

[47] 3GPP, "Study on channel model for frequencies from 0.5 to $100 \mathrm{GHz}$," TR 38.901 (release 14), May 2017.

[48] M. R. Akdeniz et al., "Millimeter wave channel modeling and cellular capacity evaluation," IEEE J. Sel. Areas Commun., vol. 32, no. 6, pp. 1164-1179, Jun. 2014.

[49] Y. Xing, O. Kanhere, S. Ju, and T. S. Rappaport, "Indoor Wireless Channel Properties at Millimeter Wave and Sub-Terahertz Frequencies," in Proc. IEEE GLOBECOM, Dec. 2019, pp. 1-6.

[50] E. Hyytia, H. Koskinen, P. Lassila, A. Penttinen, J. Virtamo, and J. Roszik, "Random Waypoint Model in Wireless Networks," Netw. and Algorithms: Complexity in physics and Comput. Sci., June 2005.

[51] M. R. Akdeniz, Y. Liu, S. Rangan, and E. Erkip, "Millimeter wave picocellular system evaluation for urban deployments," in 2013 IEEE Globecom Workshops (GC Wkshps), Dec. 2013, pp. 105-110.

[52] 3GPP, “TS 22.261, Service requirements for next generation new services and markets (Release 15),” Jul. 2018.

[53] 3GPP, “TR 38.214,, NR - Physical layer procedures for data - (release 15) document,” Jun. 2018.

[54] — _ “TR 38.900,study on channel model for frequency spectrum above $6 \mathrm{GHz}$ release," Jun. 2018.

[55] J. Campos, "Understanding the 5G NR Physical Layer," Nov. 2017.

[56] P. Mogensen et al., "LTE Capacity Compared to the Shannon Bound," in Proc. IEEE Veh. Technol. Conf. (VTC-Spring), vol. 1, May 2007, pp. $1234-1238$.

[57] "6G: The next hyper - connected experience for all," White paper, Samsung Research. [Online]. Available: https://cdn.codeground.org/nsr/downloads/researchareas/6G\%20Vision.pdf

[58] W. Saad, M. Bennis, and M. Chen, "A vision of 6G wireless systems: Applications, trends, technologies, and open research problems," IEEE Netw., vol. 34, no. 3, pp. 134-142, 2020.

[59] T. Bai, A. Alkhateeb, and R. W. Heath, "Coverage and capacity of millimeter-wave cellular networks," IEEE Commun. Mag., vol. 52, no. 9, pp. 70-77, Sep. 2014.

[60] 3GPP, “3GPP TS 38.331. NR; Radio Resource Control (RRC) protocol specification,” Oct. 2019. 